\title{
Search for high mass dijet resonances with a new background prediction method in proton-proton collisions at $\sqrt{s}=13 \mathrm{TeV}$
}

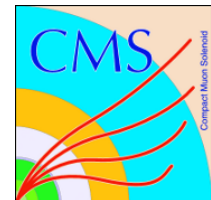

\section{The CMS collaboration}

E-mail: cms-publication-committee-chair@cern.ch

ABSTRACT: A search for narrow and broad resonances with masses greater than $1.8 \mathrm{TeV}$ decaying to a pair of jets is presented. The search uses proton-proton collision data at $\sqrt{s}=13 \mathrm{TeV}$ collected at the LHC, corresponding to an integrated luminosity of $137 \mathrm{fb}^{-1}$. The background arising from standard model processes is predicted with the fit method used in previous publications and with a new method. The dijet invariant mass spectrum is well described by both data-driven methods, and no significant evidence for the production of new particles is observed. Model independent upper limits are reported on the production cross sections of narrow resonances, and broad resonances with widths up to $55 \%$ of the resonance mass. Limits are presented on the masses of narrow resonances from various models: string resonances, scalar diquarks, axigluons, colorons, excited quarks, color-octet scalars, $\mathrm{W}^{\prime}$ and $\mathrm{Z}^{\prime}$ bosons, Randall-Sundrum gravitons, and dark matter mediators. The limits on narrow resonances are improved by 200 to $800 \mathrm{GeV}$ relative to those reported in previous CMS dijet resonance searches. The limits on dark matter mediators are presented as a function of the resonance mass and width, and on the associated coupling strength as a function of the mediator mass. These limits exclude at $95 \%$ confidence level a dark matter mediator with a mass of $1.8 \mathrm{TeV}$ and width $1 \%$ of its mass or higher, up to one with a mass of $4.8 \mathrm{TeV}$ and a width $45 \%$ of its mass or higher.

Keywords: Beyond Standard Model, Hadron-Hadron scattering (experiments)

ARXIV EPRINT: 1911.03947 


\section{Contents}

1 Introduction 1

2 The CMS detector 2

3 Jet reconstruction and event selection $\quad 2$

4 Data and simulation comparison 4

5 Background prediction methods $\quad 6$

6 Limits on the resonance cross section, mass, and coupling 10

$\begin{array}{lll}6.1 & \text { Narrow resonances } & 12\end{array}$

$\begin{array}{lll}6.2 \text { Broad resonances } & 16\end{array}$

$\begin{array}{lll}7 & \text { Summary } & 18\end{array}$

$\begin{array}{ll}\text { The CMS collaboration } & 25\end{array}$

\section{Introduction}

New particles that decay to pairs of jets and appear as dijet resonances arise in a variety of models. String resonances [1,2] originate from the Regge excitations of quarks and gluons. Scalar diquarks [3] are predicted by a grand unified theory based on the $E_{6}$ gauge symmetry group. Mass-degenerate excited quarks $\left(\mathrm{q}^{*}\right)[4,5]$ appear in quark compositeness models. Axigluons and colorons, axial-vector and vector particles, are expected in the chiral color [6, 7] and the flavor-universal coloron [7, 8] models, respectively. Color-octet scalars [9] appear in dynamical electroweak (EW) symmetry breaking models, such as technicolor. New gauge bosons $\left(\mathrm{W}^{\prime}\right.$ and $\left.\mathrm{Z}^{\prime}\right)$ can exist with standard model (SM) like or leptophobic couplings [10]. Randall-Sundrum (RS) gravitons are predicted in the RS model of extra dimensions [11]. Dark matter (DM) mediators arise from an interaction between quarks and DM [12-15]. The natural width, $\Gamma$, of a new particle increases with its coupling strength to other states, and may vary from narrow to broad, as defined in comparison to the experimental resolution.

This paper describes a model-independent search for a narrow or broad $s$-channel dijet resonance with a mass above $1.8 \mathrm{TeV}$, in proton-proton $(\mathrm{pp})$ collisions at $\sqrt{s}=13 \mathrm{TeV}$. This search uses data corresponding to an integrated luminosity of $137 \mathrm{fb}^{-1}$ collected in 20162018 with the CMS detector at the LHC. Similar searches have been published previously by the ATLAS and CMS Collaborations at $\sqrt{s}=13 \mathrm{TeV}$ [16-21], $8 \mathrm{TeV}$ [22-25], and $7 \mathrm{TeV}$ [2632] using strategies reviewed in ref. [33]. Results of the search are interpreted using as 
benchmarks the models described above. As no excess above the SM was observed, we set limits on the production cross sections of new particles decaying to the parton pairs qq (or q $\bar{q}$ ), qg, and gg. We then use these limits to constrain the benchmark models, with the same choices of parameters as those that were used in the most recent CMS search [17], which used data corresponding to an integrated luminosity of $36 \mathrm{fb}^{-1}$. In the color-octet scalar model, the squared anomalous coupling $k_{\mathrm{s}}^{2}=1 / 2$ [34] is used. For the RS graviton model, the value of the dimensionless coupling $k / \bar{M}_{\mathrm{Pl}}$ is chosen to be 0.1 , where $k$ is the curvature scale in the 5-dimensional anti de Sitter space and $\bar{M}_{\mathrm{Pl}}$ is the reduced Planck scale defined as $M_{\mathrm{Pl}} / \sqrt{8 \pi}$. For the DM mediator, we follow the recommendations of ref. [15] on model choice and coupling values. We use a simplified model [14] of a spin-1 mediator decaying only to quark-antiquark $(\mathrm{q} \overline{\mathrm{q}})$ and DM particle pairs, with an unknown mass $m_{\mathrm{DM}}$, and with a universal quark coupling $g_{\mathrm{q}}=0.25$ and a DM coupling $g_{\mathrm{DM}}=1.0$.

Similar to past searches, and for dijet mass $\left(m_{\mathrm{jj}}\right)$ greater than $1.5 \mathrm{TeV}$, the main background from quantum chromodynamics (QCD) multijet production is predicted by fitting the $m_{\mathrm{jj}}$ distribution with an empirical functional form. For $m_{\mathrm{jj}}>2.4 \mathrm{TeV}$, a new datadriven method is introduced, which predicts the background from a control region where the pseudorapidity separation of the two jets, $|\Delta \eta|$, is large. This new "ratio method" yields smaller systematic uncertainties when performed in the same dijet mass range as the "fit method", and the sensitivity for broad resonance searches is improved by up to a factor of two depending on the resonance width and mass. In addition, the total integrated luminosity for this search is roughly a factor of four larger than that used by the previous CMS search [17], so the sensitivity of both narrow and broad resonance searches has also increased by up to an additional factor of two.

\section{The CMS detector}

A detailed description of the CMS detector and its coordinate system, including definitions of the azimuthal angle $\phi$ and pseudorapidity $\eta$, is given in ref. [35]. The central feature of the CMS apparatus is a superconducting solenoid of $6 \mathrm{~m}$ internal diameter providing an axial magnetic field of $3.8 \mathrm{~T}$. Within the solenoid volume are located the silicon pixel and strip tracker $(|\eta|<2.4)$, and the barrel and endcap calorimeters $(|\eta|<3.0)$, where these latter detectors consist of a lead tungstate crystal electromagnetic calorimeter and a brass and scintillator hadron calorimeter. An iron and quartz-fiber hadron calorimeter is located in the forward region $(3.0<|\eta|<5.0)$, outside the solenoid volume. The muon detection system covers $|\eta|<2.4$ with up to four layers of gas-ionization chambers installed outside the solenoid and embedded in the layers of the steel flux-return yoke.

\section{Jet reconstruction and event selection}

A particle-flow (PF) event algorithm aims to reconstruct and identify each individual particle in an event, with an optimized combination of information from the various elements of the CMS detector [36]. Particles are classified as muons, electrons, photons, charged hadrons, or neutral hadrons. To reconstruct jets, the anti- $k_{\mathrm{T}}$ algorithm $[37,38]$ is used 
with a distance parameter of 0.4 , as implemented in the FASTJET package [39]. At least one reconstructed vertex is required. Charged PF candidates not originating from the primary vertex are removed prior to the jet finding. The candidate vertex with the largest value of summed physics-object $p_{\mathrm{T}}^{2}$, where $p_{\mathrm{T}}$ is the transverse momentum, is taken to be the primary pp interaction vertex. The physics objects are the jets, clustered using the jet finding algorithm mentioned above, with the tracks assigned to candidate vertices as inputs, and the associated missing transverse momentum, taken as the negative vector sum of the $p_{\mathrm{T}}$ of those jets. For jets, an event-by-event correction based on jet area $[40,41]$ is applied to the jet energy to remove the estimated contribution from additional collisions in the same or adjacent bunch crossings (pileup).

Events are selected using a two-tier trigger system [42]. Events satisfying loose jet requirements at the first-level (L1) trigger are examined by the high-level trigger (HLT) system. Single-jet triggers that require a jet in the event to exceed a predefined $p_{\mathrm{T}}$ threshold are used. Triggers that require $H_{\mathrm{T}}$ to exceed a threshold, where $H_{\mathrm{T}}$ is the scalar sum of jet $p_{\mathrm{T}}$ for all jets in the event with $p_{\mathrm{T}}>30 \mathrm{GeV}$ and $|\eta|<3.0$, are also used. The HLT requires: $H_{\mathrm{T}}>1050 \mathrm{GeV}$ or at least one jet reconstructed with an increased distance parameter of 0.8 and $p_{\mathrm{T}}>550 \mathrm{GeV}$.

The jet momenta and energies are corrected using calibration factors obtained from simulation, test beam results, and pp collision data at $\sqrt{s}=13 \mathrm{TeV}$. The methods described in ref. [41] are used and all in-situ calibrations are obtained from the current data. Jets are required to have $p_{\mathrm{T}}>30 \mathrm{GeV}$ and $|\eta|<2.5$. The two jets with the largest $p_{\mathrm{T}}$ are defined as the leading jets. Jet identification criteria are applied to remove spurious jets associated with the calorimeter noise as well as those associated with muon and electron candidates that are either misreconstructed or isolated [43]. For all jets, we require that the neutral hadron and photon energies are less than $90 \%$ of the total jet energy. For jets within the fiducial tracker coverage, we additionally require the jet to have nonzero charged-hadron energy, and electron and muon energies to be less than $90 \%$ and $80 \%$ of the total jet energy respectively. An event is rejected if either of the two leading jets fails these jet identification criteria.

Each of the two leading jets is formed into a "wide jet" using an algorithm introduced for previous CMS dijet searches in ref. [23]. This wide-jet algorithm, designed for dijet resonance event reconstruction, reduces the sensitivity of the analysis to gluon radiation (g) from the final-state partons. The two leading jets are used as seeds and the four-vectors of all other jets, if within a distance defined as $\sqrt{(\Delta \eta)^{2}+(\Delta \phi)^{2}}<1.1$, are added to the nearest leading jet to obtain two wide jets, which then form the dijet system. The dijet mass is then found as the invariant mass of the system of these two wide jets. The widejet algorithm thereby collects hard-gluon radiation found near the leading two final-state partons, in order to improve the dijet mass resolution.

The background from $t$-channel dijet events has the same angular distribution as Rutherford scattering, approximately proportional to $1 /[1-\tanh (|\Delta \eta| / 2)]^{2}$, which peaks at large values of $|\Delta \eta|$, the pseudorapidity separation of the two jets. The signal region (SR) is defined by requiring $|\Delta \eta|<1.1$, which maximizes the search sensitivity for isotropic 
decays of dijet resonances in the presence of QCD dijet background. For the ratio method of estimating the background, two control regions (CRs) are defined from events within $1.1<|\Delta \eta|<2.6$. The primary control region, $\mathrm{CR}_{\text {high }}$, which contains events that satisfy $1.5<|\Delta \eta|<2.6$, is used to predict the main QCD background in the SR. The secondary

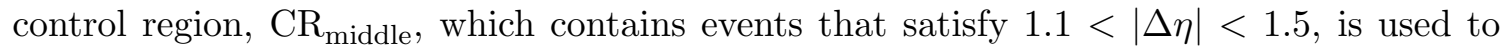
constrain theoretical and experimental systematic uncertainties. The $\mathrm{CR}_{\text {high }}$ is defined such that it has four to five times more background events than the SR, and at the same time fewer signal events by a factor of two. The SR is used to search for the presence of resonances and to estimate the QCD background for the fit method.

Events with $m_{\mathrm{jj}}>1.5 \mathrm{TeV}$ are selected offline, for which the $|\Delta \eta|$ between the two jets is in the interval $|\Delta \eta|<2.6$, where the dijet mass and $|\Delta \eta|$ are reconstructed using wide jets. For this selection the combined L1 trigger and HLT was found to be fully efficient, as measured using a sample acquired with an independent trigger requiring at least one muon with $p_{\mathrm{T}}>50 \mathrm{GeV}$ at the HLT. The $|\Delta \eta|<1.1$ requirement makes the trigger efficiency increase sharply and plateau at a value of $100 \%$ for relatively low values of dijet mass. This is because the jet $p_{\mathrm{T}}$ threshold of the trigger at a fixed dijet mass is more easily satisfied at low $|\Delta \eta|$, as seen by the approximate relation $m_{\mathrm{jj}} \approx 2 p_{\mathrm{T}} \cosh (|\Delta \eta| / 2)$. Hence, the trigger efficiency reaches $100 \%$ in the SR at a lower value of dijet mass $(1.5 \mathrm{TeV})$ than in both CRs $(2.4 \mathrm{TeV})$. Therefore the fit method is used for $m_{\mathrm{jj}}>1.5 \mathrm{TeV}$ and the ratio method, which requires data from the CRs with $100 \%$ trigger efficiency, is used for $m_{\mathrm{jj}}>2.4 \mathrm{TeV}$.

\section{Data and simulation comparison}

As the dominant background for this analysis is expected to be the QCD production of two or more jets, the selected dijet data are compared with QCD predictions. The predictions come from 270 million simulated events produced by the PYTHIA 8.205 [44] program with the CUETP8M1 tune $[45,46]$ using the parton distribution function (PDF) set NNPDF2.3LO [47], including a GEANT4-based [48] simulation of the CMS detector. The data-over-simulation ratio of event yields is 0.94 . This search uses the signal shapes of narrow and broad resonances presented in ref. [17], which are also from a PYTHIA simulation.

The dijet $|\Delta \eta|$ separation between the two wide jets is shown in figure 1 . The data distribution shows that dijet production is dominated by $t$-channel parton exchange, as predicted by QCD, with a production rate that increases with increasing $|\Delta \eta|$. By contrast, most $s$-channel signals from dijet resonances decrease with increasing $|\Delta \eta|$, as the signal shown does. Figure 1 shows the division of the $|\Delta \eta|$ distribution into the signal and control regions.

Figure 2 shows, for both data and the QCD background, the dijet mass spectra in the signal and control regions, which fall steeply and smoothly as a function of dijet mass. The observed dijet mass distributions are compared to the QCD background prediction from PYTHIA, which simulates processes at leading order (LO).

We inspect the characteristics of the 23 events with $m_{\mathrm{jj}}>7 \mathrm{TeV}$, to determine if they have the two-jet topology typical of the QCD background and to check for the presence of detector and reconstruction pathologies, and we find the one unusual event, shown in 


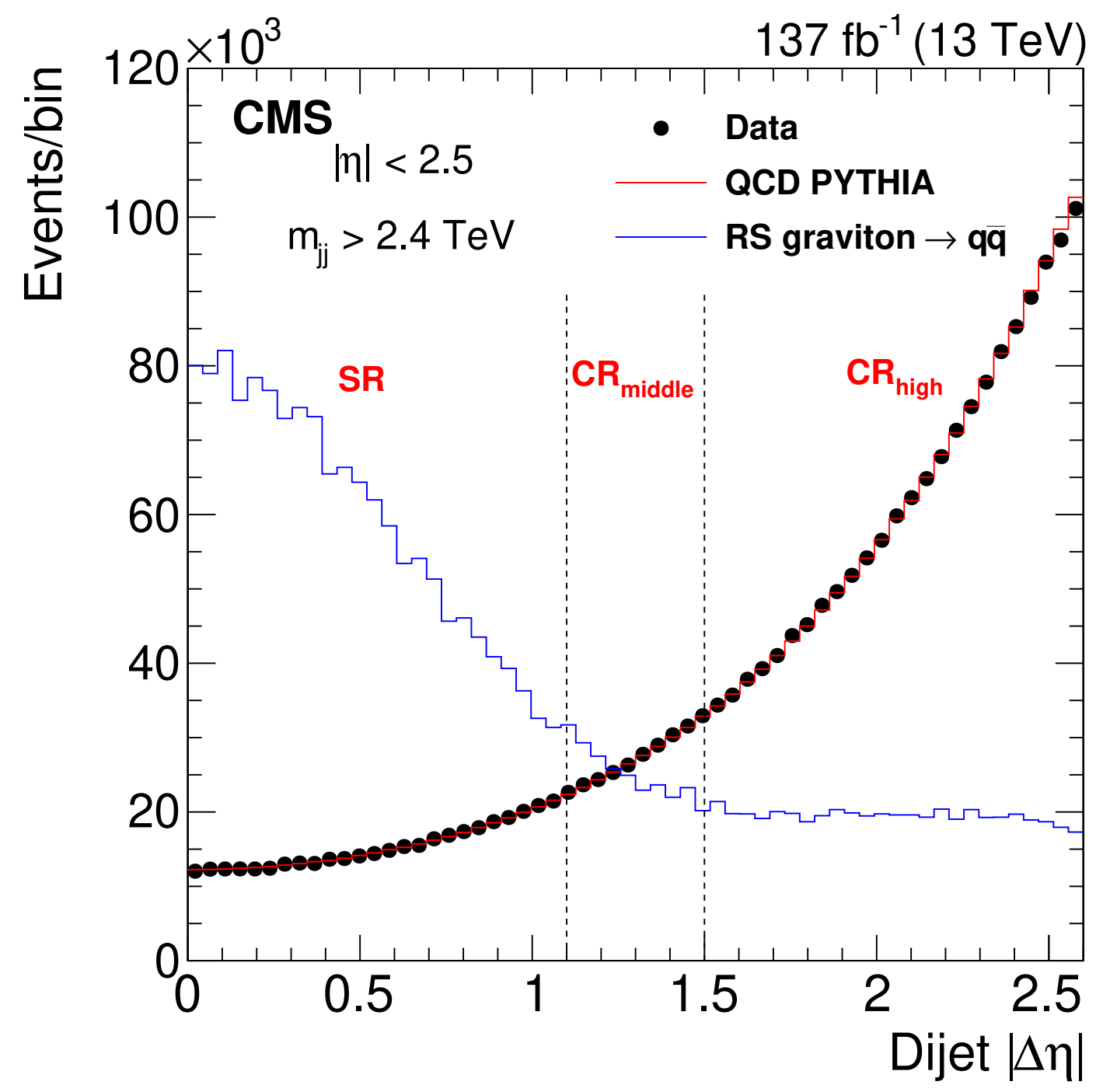

Figure 1. The pseudorapidity separation between the two wide jets for the signal and control regions. Data (black points) are compared to QCD predictions from the PYTHIA MC with detector simulation (red histogram) normalized to data. A signal from an RS graviton decaying into a q $\bar{q}$ pair is also shown (blue histogram) normalized to data.

figure 3. This event is the one with the second highest dijet mass, $8 \mathrm{TeV}$, and is unusual because it is composed of four jets, in two pairs, which are combined into the two wide jets. It is also unusual because the wide jet mass, equal to the pair mass of the jets, has the same value $1.8 \mathrm{TeV}$ for each of the two wide jets. The leading wide jet has a $p_{\mathrm{T}}$ of $3.5 \mathrm{TeV}$, and the other wide jet has a $p_{\mathrm{T}}$ of $3.4 \mathrm{TeV}$. The wide jets are back-to-back in azimuthal angle $(\Delta \phi=3.1)$ and nearby in pseudorapidity $(|\Delta \eta|=0.4)$. Each one of the two wide jets is composed of two jets with cone size 0.4 , with $p_{\mathrm{T}}, \eta$, and $\phi$ values as shown in figure 3 .

The possibility that this event originates from a resonance decaying to a pair of dijet resonances has been recently explored in a phenomenology paper [49]. 


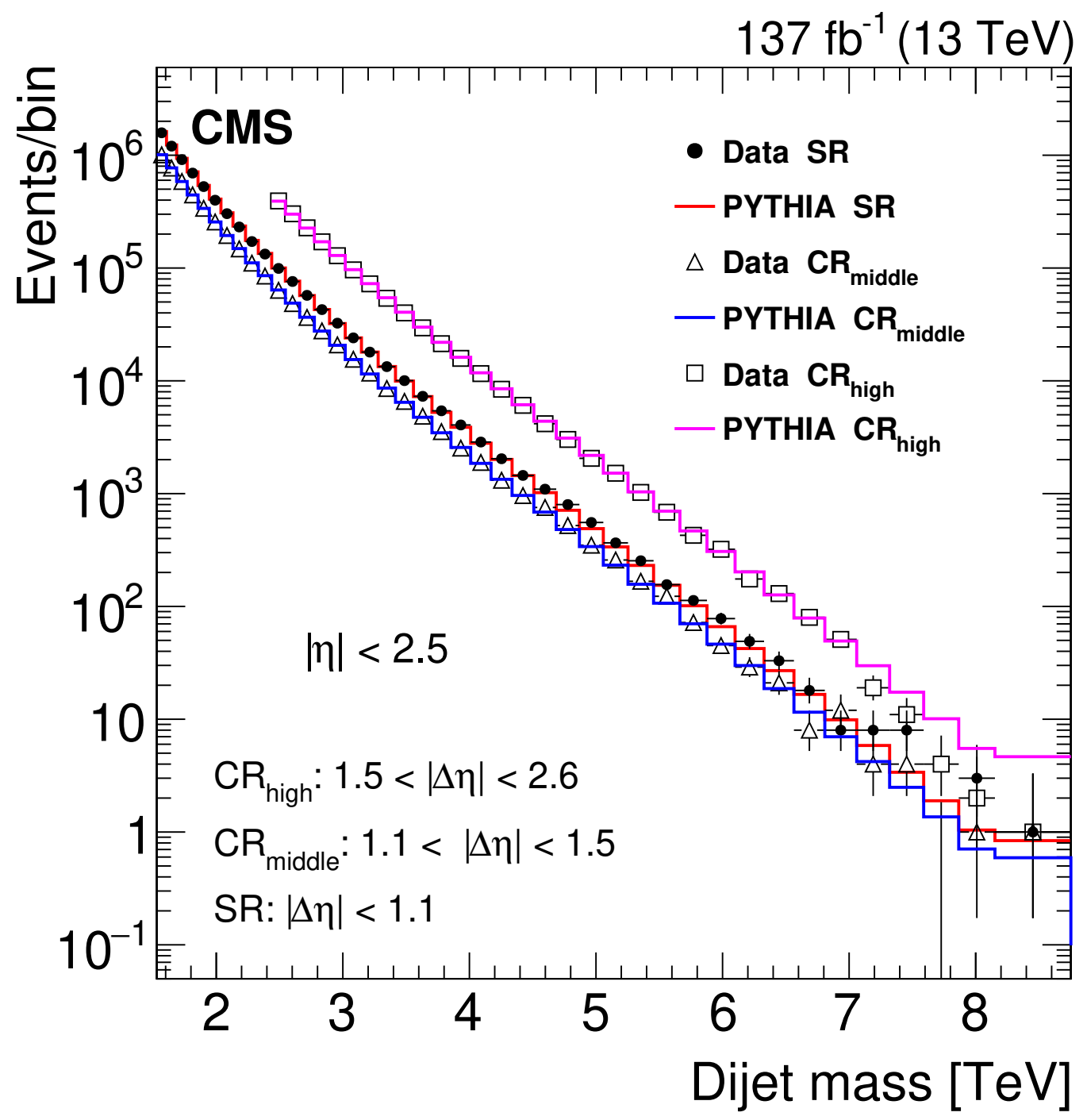

Figure 2. The dijet mass spectra of the data and PYTHIA simulation in the signal region at low $|\Delta \eta|$ (black points and red histogram), control region at middle $|\Delta \eta|$ (triangles and blue histogram), and control region at high $|\Delta \eta|$ (squares and magenta histogram). The simulation is normalized to data.

\section{$5 \quad$ Background prediction methods}

In the fit method, utilized here and in previous dijet resonance searches $[17,19-32,50]$, the main background in the SR coming from QCD is parametrized with an empirical function of the form

$$
\frac{\mathrm{d} \sigma}{\mathrm{d} m_{\mathrm{jj}}}=\frac{P_{0}(1-x)^{P_{1}}}{x^{P_{2}+P_{3} \ln (x)}}
$$

where $x=m_{\mathrm{jj}} / \sqrt{s}$, and $P_{0}, P_{1}, P_{2}$, and $P_{3}$ are four free parameters. The search for resonances proceeds with fitting the dijet mass distribution in the SR using this background parametrization and the signal template obtained from simulation, a procedure denoted as a signal plus background fit. In this fit, $P_{0}, P_{1}, P_{2}$, and $P_{3}$ are treated as freely 


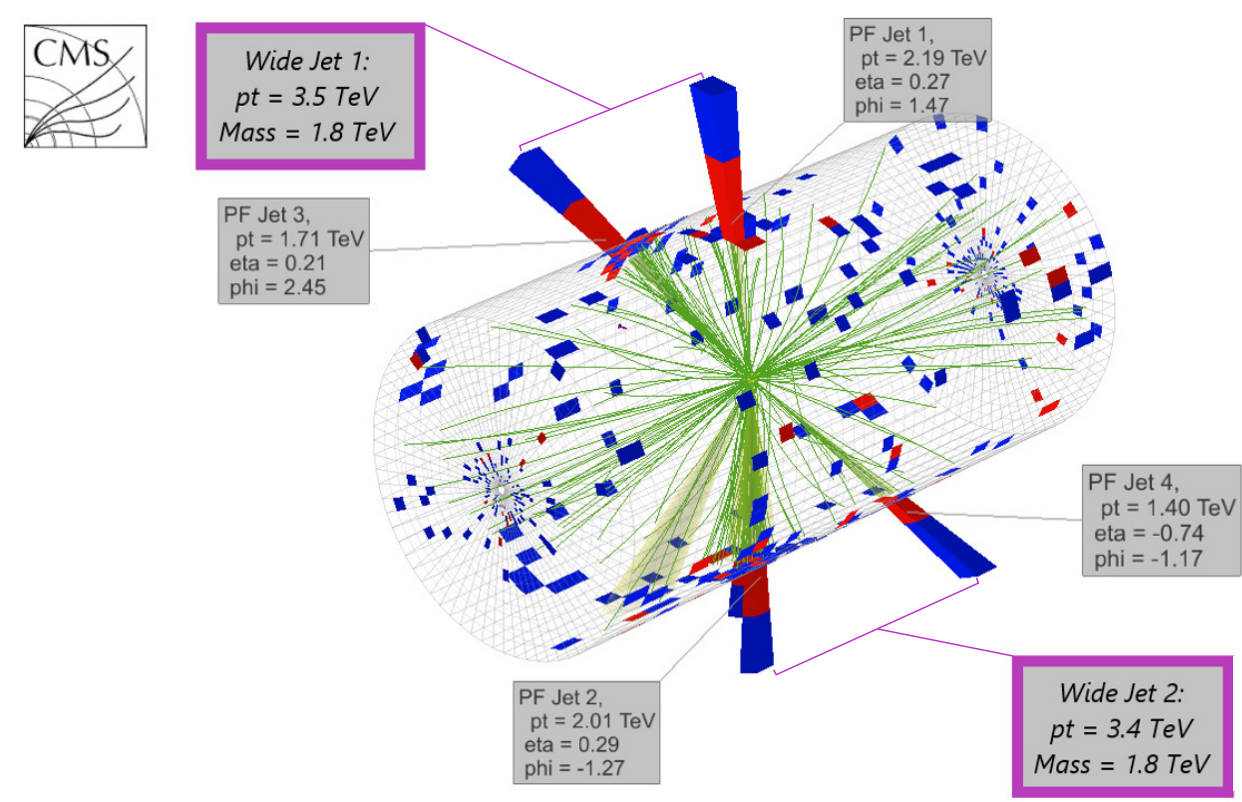

Figure 3. Three-dimensional display of the event with the second-highest dijet invariant mass of $8 \mathrm{TeV}$. The display shows the energy deposited in the electromagnetic (red) and hadronic (blue) calorimeters and the reconstructed tracks of charged particles (green). The grouping of four observed jets into two wide jets (purple) is discussed in the text.

floating nuisance parameters. In order to examine the compatibility of the data with the background-only description, and the quality of the background prediction, a fit under only the background hypothesis, denoted as a background-only fit, is also performed. The chi-square per the number of degrees of freedom of the background-only fit is $\chi^{2} / \mathrm{NDF}=$ $36.63 / 38$, as shown in figure 5 .

The ratio method is a data-driven prediction of the QCD background in the SR, obtained by multiplying the data in $\mathrm{CR}_{\text {high }}$ by a mass-dependent transfer factor determined from the simulated angular distribution of QCD dijet production. The transfer factor is the ratio, $R$, between the simulated dijet mass distribution of background events in the SR and $\mathrm{CR}_{\text {high }}$. The method makes use of the following definitions:

$$
\begin{aligned}
N(i)_{\mathrm{SR}}^{\text {Prediction }} & =R\left(m_{\mathrm{jj}} / \sqrt{s}\right) N(i)_{\mathrm{CR}_{\mathrm{high}}}^{\mathrm{Data}}, \\
R\left(m_{\mathrm{jj}} / \sqrt{s}\right) & =C\left(m_{\mathrm{jj}} / \sqrt{s}\right) N(i)_{\mathrm{SR}}^{\mathrm{Sim}} / N(i)_{\mathrm{CR}_{\mathrm{high}}}^{\operatorname{Sim} .}
\end{aligned}
$$

where $N(i)$ is the number of events in a given bin, $i$, of dijet mass and $C\left(m_{\mathrm{jj}} / \sqrt{s}\right)$ is a correction to the simulated transfer factor. This correction is required because, as seen in the upper right panel of figure 4, differences are present between data and the simulation using PYTHIA. These are due to both theoretical and experimental effects. The theoretical effects arise because the PYTHIA simulation uses a QCD calculation at LO, and higher order QCD corrections have some effect, and so do missing EW corrections. Figure 4 shows, with a smaller sample of events, that a better agreement is obtained when these corrections are included, by generating events at next-to-leading order (NLO) in QCD with POWHEG 
v2.0 [51-53] and incorporating an estimate of EW effects [54]. Experimental effects include differences between data and simulation at higher jet pseudorapidities outside the barrel calorimeter region $(|\eta|>1.3)$. The higher-order QCD and EW effects, and the differences between data and simulation at higher jet pseudorapidity values, produce a similar effect on the shape of the transfer factor, affecting mainly the high dijet mass region. We correct the simulated transfer factor to include these effects in a data-driven way, using the second control region, $\mathrm{CR}_{\text {middle, }}$ which is a $|\Delta \eta|$ sideband to the $\mathrm{SR}$. This second control region contains dijet events with values of jet pseudorapidity very similar to those in the SR, and has a very small signal contamination. As such, the dijet mass distribution of this control region is very similar to that of the SR, and the differences between data and simulation in this control region are caused by similar theoretical and experimental effects as observed in the SR. Hence, this second CR allows the definition of an auxiliary transfer factor, $R_{\text {aux }}$, shown in eq. (5.2).

$$
R_{\text {aux }}(i)=N(i)_{\mathrm{CR}_{\text {middle }}} / N(i)_{\mathrm{CR}_{\text {high }}} .
$$

We then estimate the correction, $C$, to the main transfer factor, $R$, by performing a fit to the data-over-simulation ratio of $R_{\text {aux }}$ (eq. (5.3)):

$$
R_{\text {aux }}^{\text {Data }} / R_{\text {aux }}^{\text {Sim. }}
$$

with the correction parametrized using a two-parameter empirical function, shown in eq. (5.4).

$$
C\left(m_{\mathrm{jj}} / \sqrt{s}\right)=p_{0}+p_{1}\left(m_{\mathrm{jj}} / \sqrt{s}\right)^{3} .
$$

The data to simulation ratios of the two transfer factors, $R_{\text {aux }}$ and $R$, along with their background-only fits, performed separately in order to examine their compatibility, are shown in the lower panels of figure 4 and agree to within their uncertainty at $95 \%$ confidence level (CL). Specifically, the values of the parameters and their statistical uncertainties from the background-only fits of the data to simulation ratios of $R_{\text {aux }}$ are $p_{0}=0.977 \pm 0.004$ and $p_{1}=2.07 \pm 0.33$, and of $R$ are $p_{0}=0.972 \pm 0.004$ and $p_{1}=2.52 \pm 0.28$, and are entirely compatible. This agreement is expected given that the events in $\mathrm{CR}_{\text {middle }}$ and SR have, by construction, very similar jet $\eta$ and $m_{\mathrm{jj}}$ distributions. Parameters $p_{0}$ and $p_{1}$ are treated as free nuisance parameters in the final signal plus background simultaneous fit of the $\mathrm{SR}, \mathrm{CR}_{\text {middle }}$ and $\mathrm{CR}_{\text {high }}$, taking the signal contamination in the control regions into account as described in the next paragraph. The simultaneous background-only fit yields $p_{0}=0.973 \pm 0.003$ and $p_{1}=2.38 \pm 0.23$, consistent with the separate backgroundonly fits shown in figure 4 (lower panels), and with smaller uncertainty. The systematic uncertainty in the background, for both methods, is automatically evaluated via profiling. This effectively refits for the optimal values of the background parameters, allowing them to float freely, for each value of the resonance cross section.

The signal contamination in the CRs depends on the angular distribution of the model. For the models considered in this search, the signal contamination is small compared to the background. This is because we search for dijet resonances produced in the $s$-channel annihilation of two partons, while the QCD background is predominantly a $t$-channel process. We assume the signal has the same angular distribution as a vector resonance decaying 
to $q \bar{q}$ pairs. The signal contamination is taken into account in the simultaneous fit. The change in extracted signal is negligible if the angular distribution of any of our other benchmark models is chosen instead. Our benchmark models include scalars coupled to qq or gg pairs, fermions coupled to qg pairs, vectors coupled to q $\bar{q}$ pairs, and tensors coupled to q $\bar{q}$ or gg pairs.

Detailed signal injection tests are performed to investigate the potential bias in each background prediction method, and the bias is found to be negligible when either the fit method or the ratio method is employed. The signal injection tests are performed as follows: pseudo-data distributions are generated, varying the parameters of the background prediction and injecting a signal with a cross section equal to i) zero, ii) the $95 \% \mathrm{CL}$ observed limit, and iii) two times the 95\% CL observed limit. These distributions are created for several resonance masses and widths, spanning the entire range for which results are reported. Then, the same fitting procedure followed in the analysis of the actual data is repeated for each pseudo-data distribution, and the fitted signal cross section, along with its $68 \%$ CL standard deviation, is obtained. We examine the distribution of the bias in units of standard deviations, namely the difference between the injected signal cross section and the fitted signal cross section, divided by the standard deviation of the fit. For all resonance masses, widths, and signal strengths considered, the mean bias is less than one half a standard deviation, and in the vast majority of the cases it is well below this criterion. In addition, pseudo-data distributions are generated using different empirical functional forms than the ones used in the actual data fits, and the entire procedure is repeated, again yielding negligible biases.

The ratio method is an independent approach compared to the fit method, yielding consistent results. The ratio method provides a background estimate that is derived primarily from control regions, while the fit method uses only the signal region. The ratio method also provides a background estimate that is more accurate than the fit method. This is because the ratio method fits the data with only two parameters, while the fit method requires four, and because the estimate from the ratio method is additionally constrained by the control region $\mathrm{CR}_{\text {middle }}$. The advantages of this method, as opposed to the fit method, are the following: i) it provides a background estimate independent of the signal region, which results in an independent and less biased value of the observed signal significance, ii) as the resonance width increases the ratio method has smaller background uncertainty compared to the fit method, and hence higher sensitivity. Therefore, we estimate the background using the ratio method instead of the fit method for $m_{\mathrm{jj}}>2.4 \mathrm{TeV}$.

Figure 5 shows the dijet mass spectrum, defined as the observed number of events in each bin divided by the integrated luminosity and the bin width. The bin widths depend on the dijet mass and are chosen to correspond to dijet mass resolution. The bin edges were chosen to be the same as those used by previous dijet resonances searches performed by the CMS Collaboration, as introduced in ref. [31]. Figure 5 also shows the background prediction from the fit method, compared to all data, and the background prediction from the ratio method, compared to data with $m_{\mathrm{jj}}>2.4 \mathrm{TeV}$. The $\chi^{2} / \mathrm{NDF}$ of the backgroundonly fit, masking the signal region, with the ratio method is $42.04 / 32$ as shown in figure 5 . The dijet mass spectrum is well modeled by both background prediction methods, which also agree with one another. 

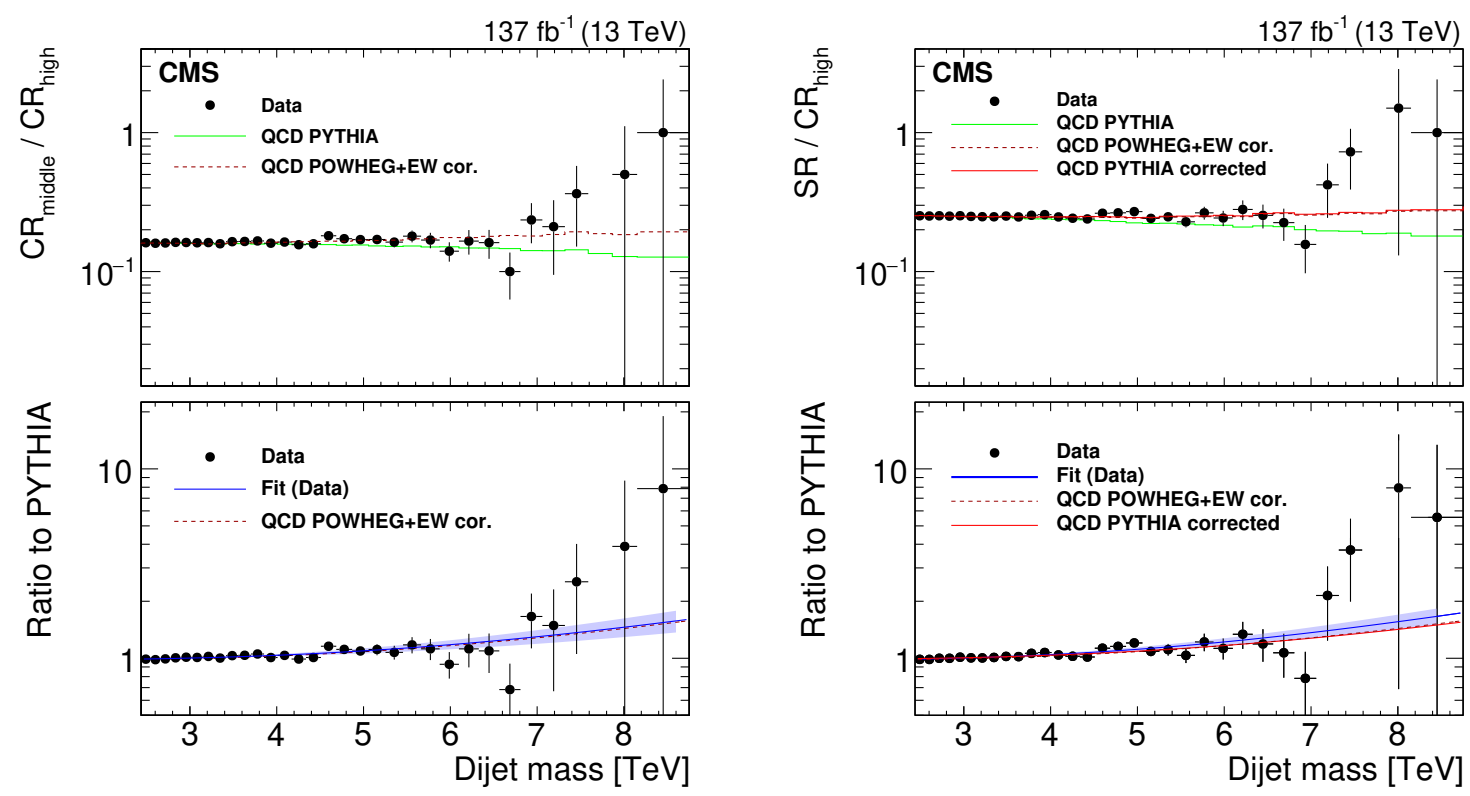

Figure 4. The ratio $R_{\text {aux }}$, the auxiliary transfer factor, calculated for data, PYTHIA, and POWHEG with electroweak corrections (left, upper panel). The double ratio of the same quantities in the upper left panel to $R_{\text {aux }}$ from PYTHIA, along with the fit of the double ratio for data with the correction function (left, lower panel). The ratio $R$, the transfer factor, calculated for data, PYTHIA, POWHEG with electroweak corrections, and corrected PYTHIA (right, upper panel). The double ratio of the same quantities in the upper right panel to $R$ from PYTHIA, along with the fit of the double ratio for data with a correction function, and corrected PYTHIA using $\mathrm{CR}_{\text {middle }}$ (right, lower panel). The fits in the two lower panels agree with each other within their uncertainty at 95\% CL (shaded bands).

\section{Limits on the resonance cross section, mass, and coupling}

We use the dijet mass spectrum from wide jets, the background parameterizations, and the dijet resonance shapes shown previously to set limits on the production cross sections of new particles decaying to the parton pairs qq (or $q \bar{q})$, qg, and gg. A separate limit is determined for each final state (qq, qg, and gg) because of the dependence of the dijet resonance shape on the types of the two final-state partons.

The dominant sources of systematic uncertainty are the jet energy scale and resolution, the integrated luminosity, and the values of the parameters within the functional form modeling the background shape in the dijet mass distribution. The uncertainty in the jet energy scale is within $2 \%$ for all values of the dijet mass and is determined from $\sqrt{s}=13 \mathrm{TeV}$ data using the methods described in ref. [41]. This uncertainty is propagated to the limits by shifting the dijet mass shape for the signal by $\pm 2 \%$. The uncertainty in the jet energy resolution translates into an uncertainty of $10 \%$ in the resolution of the dijet mass [41], and is propagated to the limits by observing the effect of increasing and decreasing by $10 \%$ the reconstructed width of the dijet mass shape for the signal. The uncertainty in the integrated luminosity is $2.5 \%$ in 2016 [55] and 2018 [56], and 2.3\% in 2017 [57], and is propagated to the normalization of the signal. Changes in the values of the parameters 

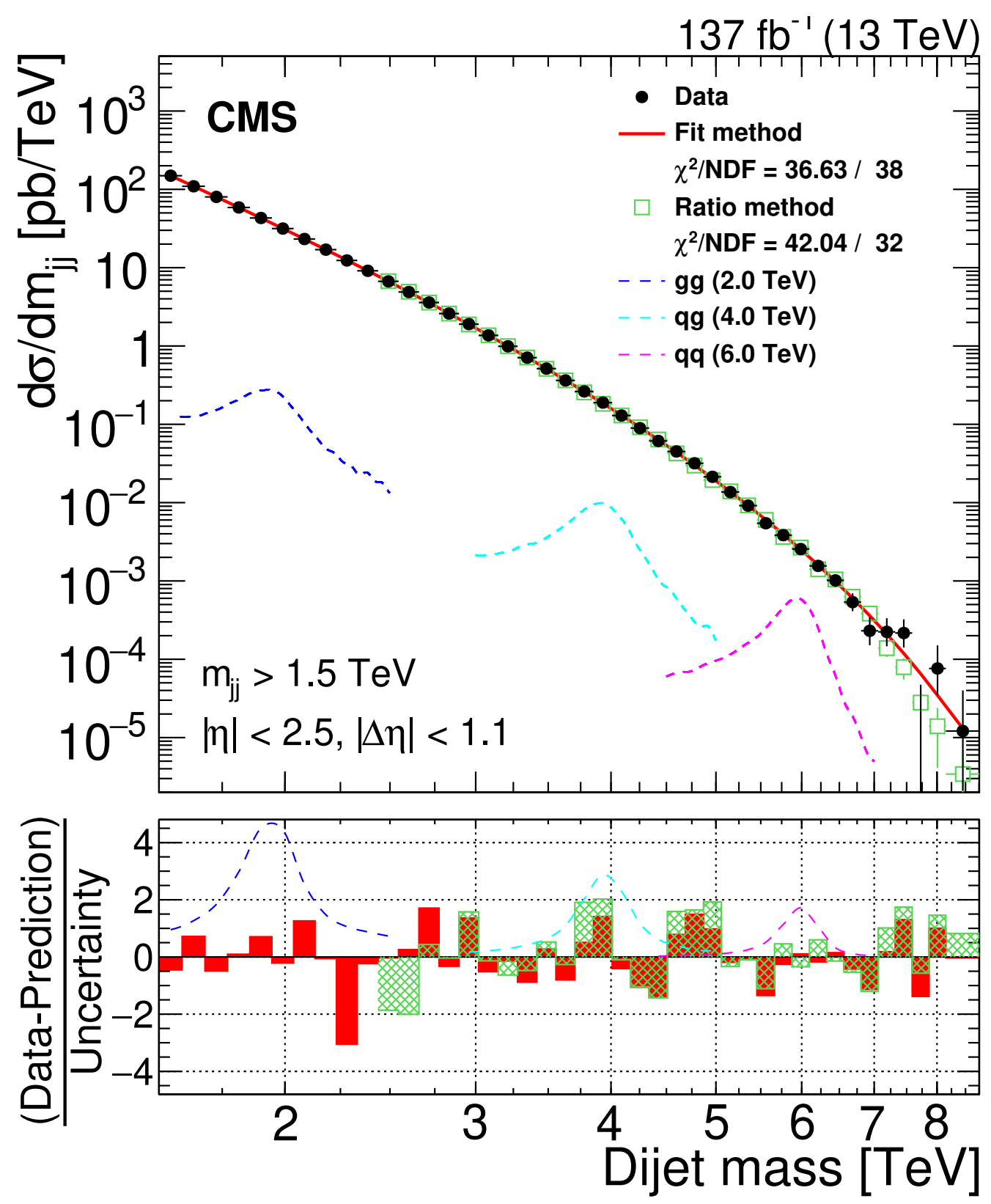

Figure 5. Dijet mass spectrum in the signal region (points) compared to a fitted parameterization of the background (solid line) and the one obtained from the control region (green squares). The lower panel shows the difference between the data and the fitted parametrization (red, solid), and the data and the prediction obtained from the control region (green, hatched), divided by the statistical uncertainty in the data, which for the ratio method includes the statistical uncertainty in the data in the control region. Examples of predicted signals from narrow gluon-gluon, quarkgluon, and quark-quark resonances are shown (dashed coloured lines) with cross sections equal to the observed upper limits at $95 \%$ CL. 
describing the background introduce a change in the signal yield, which is accounted for as a systematic uncertainty, as discussed in the next paragraph.

The modified frequentist criterion $[58,59]$ is used to set upper limits on signal cross sections, following the prescription described in refs. [60,61] using the asymptotic approximation of the test statistic. We use a multi-bin counting experiment likelihood, which is a product of Poisson distributions corresponding to different bins. We evaluate the likelihood independently at each value of resonance pole mass from 1.8 to $8.7 \mathrm{TeV}$ in $100-\mathrm{GeV}$ steps. The fit method is used to estimate the background for resonance masses from 1.8 to $2.9 \mathrm{TeV}$, and the ratio method is used for resonance masses from 3.0 to $8.7 \mathrm{TeV}$. The minimum values of resonance mass for the two methods, 1.8 and $3.0 \mathrm{TeV}$, are chosen to maintain reasonable acceptances for the minimum $m_{\mathrm{jj}}$ requirements, 1.5 and $2.4 \mathrm{TeV}$, respectively. The sources of systematic uncertainty are implemented as nuisance parameters in the likelihood model, with Gaussian constraints for the jet energy scale and resolution, and log-normal constraints for the integrated luminosity. The background systematic uncertainty, as we described previously, is automatically evaluated via profiling and decreases as the resonance mass increases.

\subsection{Narrow resonances}

Figures 6 and 7 show the model-independent observed upper limits at $95 \%$ confidence level on the product of the cross section $(\sigma)$, the branching fraction $(B)$, and the acceptance $(A)$ for narrow resonances, with the kinematic requirements $|\Delta \eta|<1.1$ for the dijet system and $|\eta|<2.5$ for each jet. The narrow resonance shapes are the ones presented and discussed in detail in a previous publication [17]. The acceptance of the minimum dijet mass requirement in each search, which fully accounts for the overall experimental acceptance, has been evaluated separately for qq, qg, and gg resonances. We include these acceptances in the determination of the limits. Figure 6 also shows the expected limits on $\sigma B A$ and their bands of uncertainty. Figure 7 shows the different limits for qq, qg, and gg resonances, which originate from differences in their line shapes. For the RS graviton, which decays to both $\mathrm{q} \overline{\mathrm{q}}$ and gg, we obtain cross section upper limits from the average, weighted by branching fraction, of the limits on quark-quark and gluon-gluon resonances.

Using the statistical methodology discussed earlier, the local significance for qq, qg, and gg resonance signals was measured from 1.8 to $8.7 \mathrm{TeV}$ in steps of $100 \mathrm{GeV}$. The significance values obtained for qq resonances are shown in figure 8 for both the ratio and the fit methods, and the significances for $\mathrm{q} g$ and $\mathrm{g} g$ resonances are the same within 0.2 standard deviations. The ratio method usually gives a larger signal significance than the fit method, because it provides a more accurate data-driven background estimate.

All upper limits presented can be compared to the parton-level predictions of $\sigma B A$, without detector simulation, to determine mass limits on new particles. The model predictions shown in figure 6 are calculated in the narrow-width approximation [33] using the CTEQ6L1 [62] parton distribution function at LO. An NLO correction factor of $K=1+$ $8 \pi \alpha_{S} / 9 \approx 1.3$ is applied to the LO predictions for the $\mathrm{W}^{\prime}$ model and $K=1+\left(4 \alpha_{S} / 6 \pi\right)(1+$ $\left.4 \pi^{2} / 3\right) \approx 1.2$ for the $\mathrm{Z}^{\prime}$ and the DM mediator models [63], where $\alpha_{S}$ is the strong coupling constant evaluated at a scale equal to the resonance mass. Similarly, for the axigluon and 

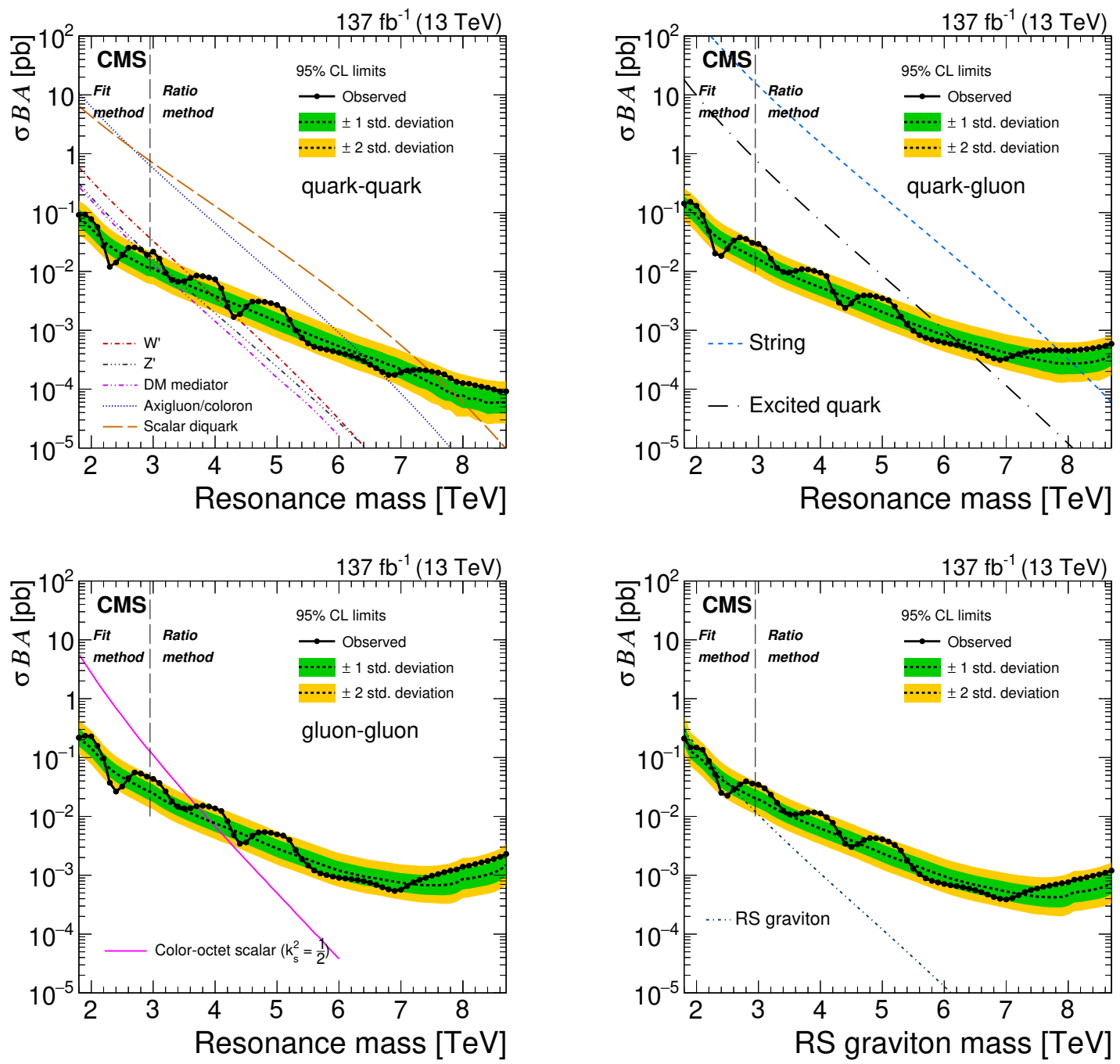

Figure 6. The observed 95\% CL upper limits on the product of the cross section, branching fraction, and acceptance for dijet resonances decaying to quark-quark (upper left), quark-gluon (upper right), gluon-gluon (lower left), and for RS gravitons (lower right). The corresponding expected limits (dashed lines) and their variations at the one and two standard deviation levels (shaded bands) are also shown. Limits are compared to predicted cross sections for string resonances [1, 2], excited quarks [4, 5], axigluons [6], colorons [8], scalar diquarks [3], color-octet scalars [9], new gauge bosons $\mathrm{W}^{\prime}$ and $\mathrm{Z}^{\prime}$ with SM-like couplings [10], DM mediators for $m_{\mathrm{DM}}=1 \mathrm{GeV}[14,15]$, and $\mathrm{RS}$ gravitons [11]. The vertical dashed line indicates the boundary between the regions where the fit method and the ratio method are used to estimate the background. 


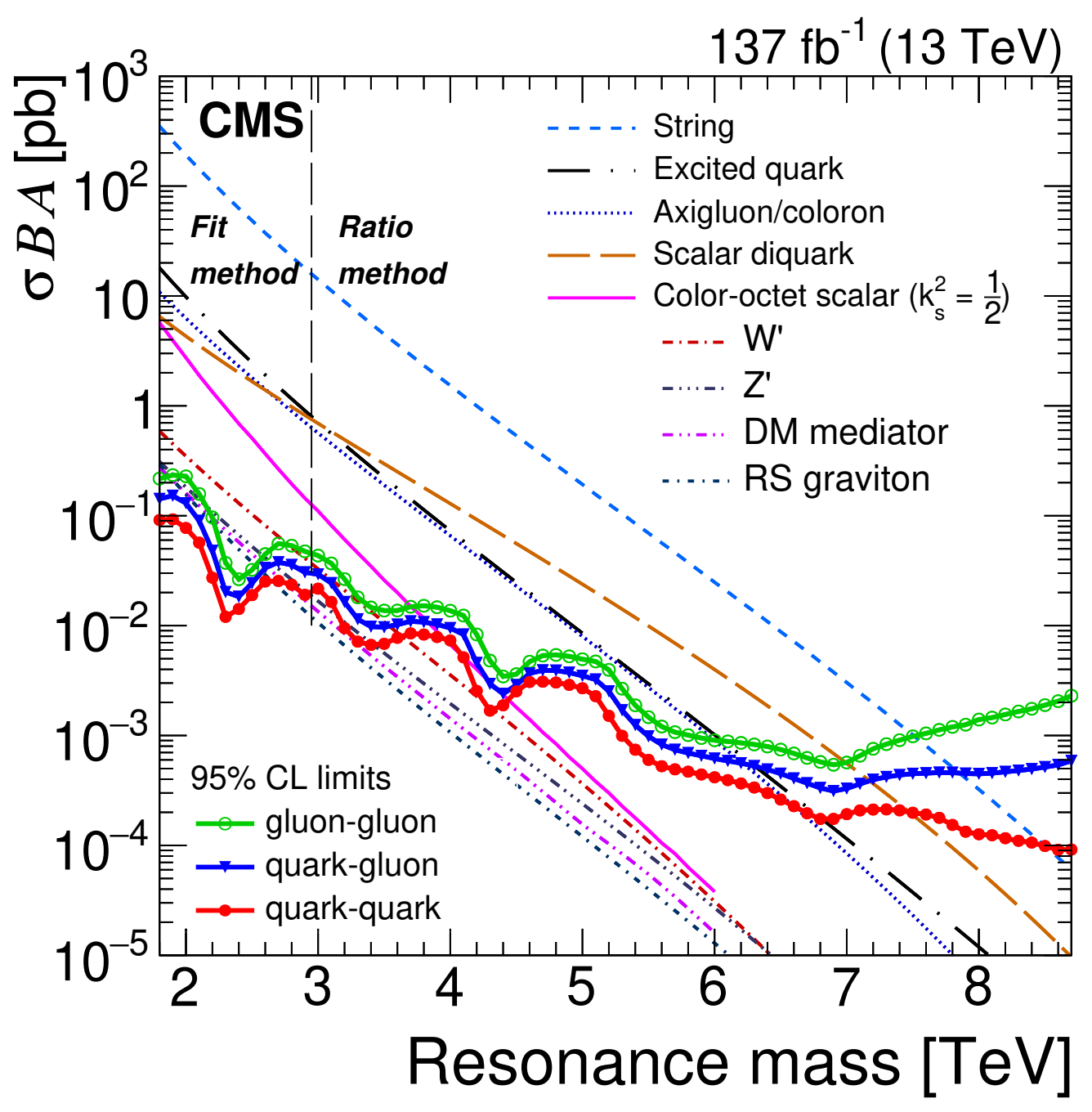

Figure 7. The observed 95\% CL upper limits on the product of the cross section, branching fraction, and acceptance for quark-quark, quark-gluon, and gluon-gluon type dijet resonances. Limits are compared to predicted cross sections for string resonances [1, 2], excited quarks [4, 5], axigluons [6], colorons [8], scalar diquarks [3], color-octet scalars [9], new gauge bosons $\mathrm{W}^{\prime}$ and $\mathrm{Z}^{\prime}$ with SM-like couplings [10], DM mediators for $m_{\mathrm{DM}}=1 \mathrm{GeV}$ [14, 15], and RS gravitons [11]. The vertical dashed line indicates the boundary between the regions where the fit method and the ratio method are used to estimate the background. 


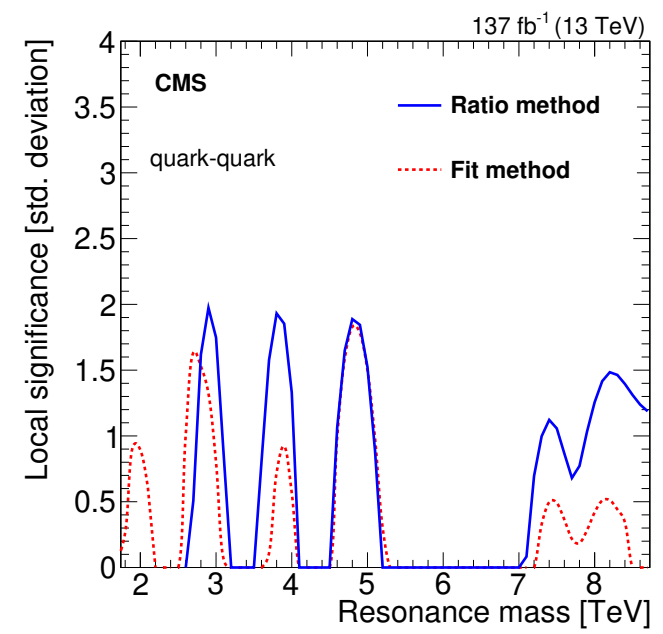

Figure 8. Local significance for a qq resonance with the ratio method (blue line) and the fit method (red dashed line).

\begin{tabular}{|lcc|}
\hline Model & Final state & Observed (expected) mass limit $[\mathrm{TeV}]$ \\
\hline String & $\mathrm{qg}$ & $7.9(8.1)$ \\
Scalar diquark & $\mathrm{qq}$ & $7.5(7.9)$ \\
Axigluon/coloron & $\mathrm{q} \overline{\mathrm{q}}$ & $6.6(6.4)$ \\
Excited quark & $\mathrm{qg}$ & $6.3(6.2)$ \\
Color-octet scalar $\left(k_{s}^{2}=1 / 2\right)$ & $\mathrm{gg}$ & $3.7(3.9)$ \\
$\mathrm{W}^{\prime}$ SM-like & $\mathrm{q} \overline{\mathrm{q}}$ & $3.6(3.9)$ \\
$\mathrm{Z}^{\prime}$ SM-like & $\mathrm{q} \overline{\mathrm{q}}$ & $2.9(3.4)$ \\
$\mathrm{RS}$ graviton $\left(k / \bar{M}_{\mathrm{Pl}}=0.1\right)$ & $\mathrm{q} \overline{\mathrm{q}}, \mathrm{gg}$ & $2.6(2.6)$ \\
$\mathrm{DM}$ mediator $\left(m_{\mathrm{DM}}=1 \mathrm{GeV}\right)$ & $\mathrm{q} \overline{\mathrm{q}}$ & $2.8(3.2)$ \\
\hline
\end{tabular}

Table 1. Observed and expected mass limits at 95\% CL from this analysis. The listed models are excluded between $1.8 \mathrm{TeV}$ and the indicated mass limit by this analysis. The SM-like $\mathrm{Z}^{\prime}$ resonance is also excluded within the mass interval between 3.1 and $3.3 \mathrm{TeV}$.

coloron models a correction factor is applied which varies between $K=1.1$ at a resonance mass of $0.6 \mathrm{TeV}$ and $K=1.3$ at $8.1 \mathrm{TeV}$ [7]. The branching fraction includes the direct decays of the resonance into the five light quarks and gluons only, excluding top quarks from the decay, although top quarks are included in the calculation of the resonance width. The acceptance is evaluated at the parton level for the resonance decay to two partons. In the case of isotropic decays, the acceptance is $A \approx 0.5$ and is independent of the resonance mass. For a given model, new particles are excluded at 95\% CL in mass regions where the theoretical prediction lies at or above the observed upper limit for the appropriate final state of figure 6 . Table 1 shows the mass limits on all benchmark models which are extended by 200 to $800 \mathrm{GeV}$ relative to those reported in previous CMS dijet resonance searches [17]. 


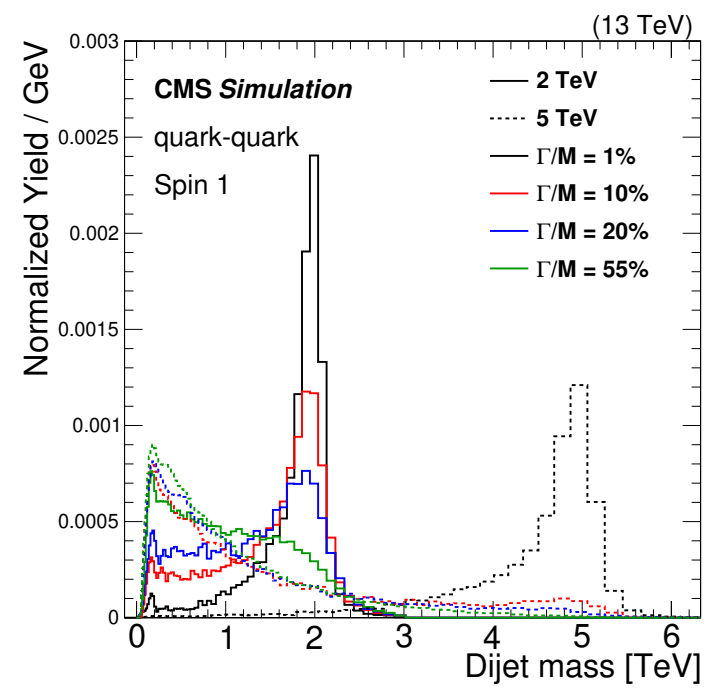

Figure 9. The reconstructed dijet mass spectra for a vector particle decaying to pairs of quarks are shown for a resonance mass of $2 \mathrm{TeV}$ (solid histogram) and $5 \mathrm{TeV}$ (dashed histogram) for various values of intrinsic width, estimated from the MADGRAPH5 and PYTHIA event generators followed by the simulation of the CMS detector response.

\subsection{Broad resonances}

We extend the search to cover broad resonances. We use spin-1 resonances decaying to quark-quark pairs with a width up to $55 \%$ of the resonance mass, $M$, as well as spin-2 resonances that decay to quark or gluon pairs with a width up to $30 \%$ of the resonance mass. This allows us to be sensitive to more models and larger couplings. The spin-1 resonance results are also used to produce limits on the universal quark coupling of a leptophobic vector mediator of interactions between quarks and DM particles, and limits for a leptophobic $Z^{\prime}$ that couples to quarks but does not couple to DM particles [12-15]. In order to be sensitive to the largest possible coupling values for these particles, the maximum value of examined widths for spin- 1 resonances is increased to $55 \%$ of the resonance mass. The additional wider signals are produced in the same way as the narrower ones, using the MAdGraPh5_aMC@NLO v. 2.3.2 [64] generator at LO, and the PYTHIA 8.205 [44] program, followed by a GEANT4-based [48] simulation of the CMS detector. For resonance widths up to $30 \%$ of their mass, the dijet mass distributions are the ones presented and discussed in detail in ref. [17]. The dijet mass distributions of both wide and narrow spin-1 resonances are shown in figure 9, and exhibit the same behavior as the ones discussed in [17].

The cross section limits in this case are presented as a function of resonance mass and width. In figure 10 we show the observed 95\% CL upper limits for various resonance widths, for spin-2 resonances modeled by an RS graviton signal in the quark-quark and gluon-gluon channels, and for spin-1 resonances in the quark-quark channel. The limits weaken as the resonance intrinsic width increases, following the characteristics of the resonance shapes. The spin- 1 resonances are significantly broader than the spin- 2 resonances. For this reason, their limits are weaker than those of the spin-2 resonances. In figure 10 the cross section 

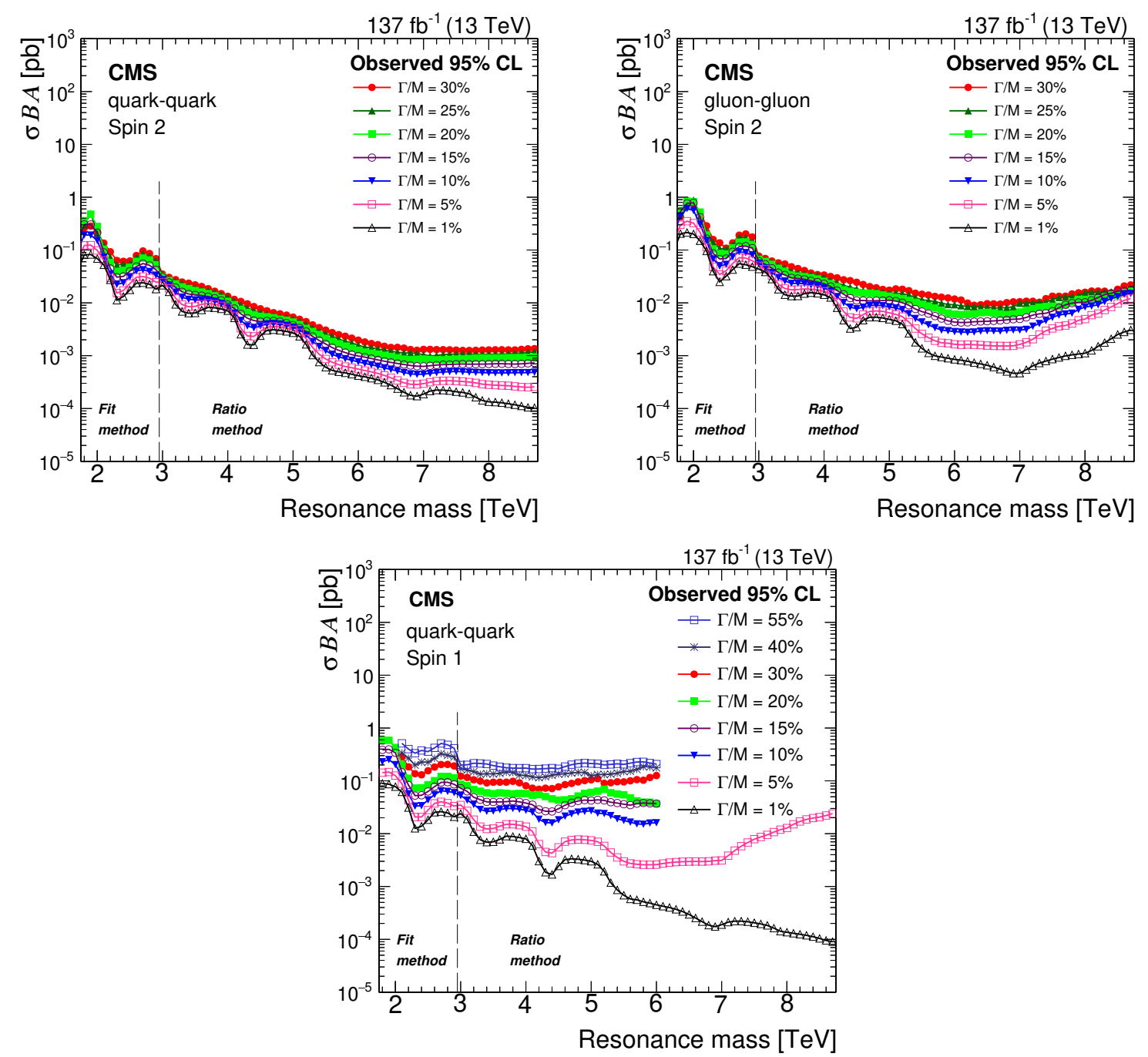

Figure 10. The observed 95\% CL upper limits on the product of the cross section, branching fraction, and acceptance for spin-2 resonances produced and decaying in the quark-quark (upper left) and gluon-gluon (upper right) channels, as well as for spin-1 resonances decaying in the quarkquark channel (lower), shown for various values of intrinsic width as a function of resonance mass. The vertical dashed line indicates the boundary between the regions where the fit method and the ratio method are used to estimate the background.

limits at very high mass for spin- 1 resonances with $\Gamma / M=5 \%$ increase as the resonance mass increases, while they decrease for $\Gamma / M=1 \%$. This is because, for resonances with widths larger than $1 \%$, the tail to low dijet mass increases significantly as the resonance mass increases, as shown in figure 9 .

The limits are presented up to a maximum resonance mass of $8.7 \mathrm{TeV}$ for most models. We do not present limits for the case of spin-1 resonances in the quark-quark channel with masses larger than $6 \mathrm{TeV}$ and $\Gamma / M>0.1$. These resonances are not part of the search because they have an exceedingly broad and high tail at low dijet mass, as described in ref. [17], which dominates the limit and produces unstable search results. The spin-1 cross 
section limits in figure 10 have been used to derive constraints on the coupling to quarks of mediators of new interactions. We consider two models of a leptophobic mediator which couples to all generations of quarks with the same universal strength. The quark coupling is denoted $g_{\mathrm{q}}^{\prime}$ in the first model, in which the mediator does not couple to DM particles, and denoted $g_{\mathrm{q}}$ in the second model, in which the mediator couples to DM particles. For each mediator mass value, the predictions for the cross section of mediator production as a function of the quark coupling are converted to predictions as a function of width. They are then compared to the spin- 1 cross section limits from figure 10 to find the excluded values of quark coupling, as a function of mass for a spin-1 resonance. Figure 11 (right) shows upper limits on the coupling $g_{\mathrm{q}}^{\prime}$ as a function of mass for our first model, also known as a leptophobic $\mathrm{Z}^{\prime}$ resonance [65] that couples only to quarks. In this model the resonance has a width

$$
\Gamma_{\mathrm{Med}}=\frac{3\left(g_{\mathrm{q}}^{\prime}\right)^{2} M_{\mathrm{Med}}}{2 \pi},
$$

where $M_{\text {Med }}$ is the resonance mass and $g_{\mathrm{q}}^{\prime}$ is the universal quark coupling, related to the coupling, $g_{B}$, of ref. [65] by $g_{\mathrm{q}}^{\prime}=g_{B} / 6$. Figure 11 (left) shows upper limits on the coupling $g_{\mathrm{q}}$ as a function of mass for our second model, also known as a DM Mediator model, which has a leptophobic spin-1 mediator that couples both to quarks and DM particles [15], and for Dirac DM with a mass $m_{\mathrm{DM}}=1 \mathrm{GeV}$ and a coupling $g_{\mathrm{DM}}=1.0$. The cross section of mediator production for $m_{\mathrm{DM}}=1 \mathrm{GeV}$ and $g_{\mathrm{DM}}=1$ is calculated with MADGRAPH5_amC@NLO [64] for mediator masses within the range $1.6<M_{\mathrm{Med}}<5.1 \mathrm{TeV}$ in $0.1 \mathrm{TeV}$ steps and for quark couplings within the range $0.1<g_{\mathrm{q}}<1.0$ in 0.1 steps. For these choices, the relationship between the total mediator width, for decays to both quark and DM particles, and $g_{\mathrm{q}}$ given in refs. $[14,15]$ simplifies to

$$
\Gamma_{\mathrm{Med}} \approx \frac{\left(18 g_{\mathrm{q}}^{2}+1\right) M_{\mathrm{Med}}}{12 \pi} .
$$

The increased sensitivity of the ratio method to wide resonances significantly improves and extends previous limits on DM mediators at large values of $\Gamma / M$. For example, for $\Gamma / M=0.45$, this search excludes DM mediators with mass less than $4.8 \mathrm{TeV}$, while the observed limit from the earlier searches was $4.0 \mathrm{TeV}$ [17].

\section{Summary}

A search for resonances decaying into a pair of jets has been performed using proton-proton collision data at $\sqrt{s}=13 \mathrm{TeV}$ corresponding to an integrated luminosity of $137 \mathrm{fb}^{-1}$. The dijet mass spectra are observed to be smoothly falling distributions of events with typically two-jet topology, although one unusual event with a four-jet topology was found at high mass. The background is predicted using two methods. The fit method uses an empirical functional form to fit the background in the signal region, defined by requiring the pseudorapidity separation of two jets in dijet $|\Delta \eta|<1.1$, while the ratio method uses two control regions at higher values of $|\Delta \eta|$ to predict the background in the signal region. The ratio 

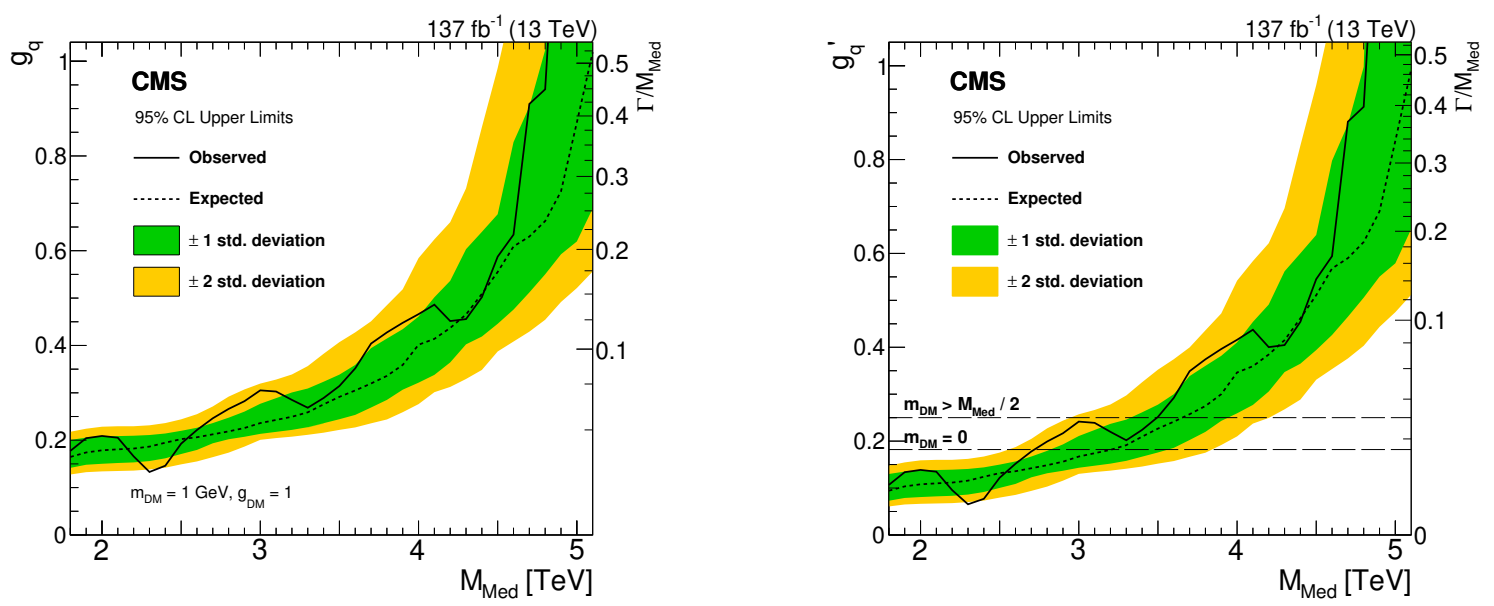

Figure 11. The 95\% CL upper limits on the universal quark coupling $g_{\mathrm{q}}$ as a function of resonance mass for a vector mediator of interactions between quarks and DM particles (left), and between quarks only (right). The dashed horizontal lines on the right plot show the coupling strength for which the cross section for dijet production in this leptophobic $\mathrm{Z}^{\prime}$ model is the same as for a DM mediator for $g_{\mathrm{q}}=0.25$. The right vertical axis shows the natural width of the mediator divided by its mass. The expected limits (dashed lines) and their variation at the one and two standard deviation levels (shaded bands) are also shown.

method is a new background prediction method, which is independent of and complementary to the fit method. No evidence for resonant particle production is observed. Generic upper limits are presented on the product of the cross section, the branching fraction, and the acceptance for narrow and broad quark-quark, quark-gluon, and gluon-gluon resonances. The limits are applied to various models of new resonances and yield the following 95\% confidence level lower limits on the resonance masses: $7.9 \mathrm{TeV}$ for string resonances, 7.5 TeV for scalar diquarks, $6.6 \mathrm{TeV}$ for axigluons and colorons, $6.3 \mathrm{TeV}$ for excited quarks, $3.7 \mathrm{TeV}$ for color-octet scalars, $3.6 \mathrm{TeV}$ for $\mathrm{W}^{\prime}$ bosons with SM-like couplings, $2.9 \mathrm{TeV}$ and between 3.1 and $3.3 \mathrm{TeV}$ for $\mathrm{Z}^{\prime}$ bosons with SM-like couplings, $2.6 \mathrm{TeV}$ for Randall-Sundrum gravitons, and $2.8 \mathrm{TeV}$ for dark matter (DM) mediators. With this search, limits on narrow resonances are improved by 200 to $800 \mathrm{GeV}$ relative to those reported in previous CMS dijet resonance searches. Limits are also presented for spin-2 resonances with intrinsic widths as large as $30 \%$ of the resonance mass, and spin- 1 resonances with intrinsic widths as large as $55 \%$ of the resonance mass. These limits are used to improve and extend the exclusions of a DM mediator to larger values of the resonance mass and coupling to quarks. In the search for broad resonances, the ratio method provides significantly enhanced sensitivity compared to the fit method, resulting in the exclusion at $95 \%$ confidence level of a DM mediator with mass less than $4.8 \mathrm{TeV}$ for a width equal to $45 \%$ of the mass, which corresponds to a coupling to quarks $g_{\mathrm{q}}=0.9$. 


\section{Acknowledgments}

We congratulate our colleagues in the CERN accelerator departments for the excellent performance of the LHC and thank the technical and administrative staffs at CERN and at other CMS institutes for their contributions to the success of the CMS effort. In addition, we gratefully acknowledge the computing centers and personnel of the Worldwide LHC Computing Grid for delivering so effectively the computing infrastructure essential to our analyses. Finally, we acknowledge the enduring support for the construction and operation of the LHC and the CMS detector provided by the following funding agencies: BMBWF and FWF (Austria); FNRS and FWO (Belgium); CNPq, CAPES, FAPERJ, FAPERGS, and FAPESP (Brazil); MES (Bulgaria); CERN; CAS, MoST, and NSFC (China); COLCIENCIAS (Colombia); MSES and CSF (Croatia); RPF (Cyprus); SENESCYT (Ecuador); MoER, ERC IUT, PUT and ERDF (Estonia); Academy of Finland, MEC, and HIP (Finland); CEA and CNRS/IN2P3 (France); BMBF, DFG, and HGF (Germany); GSRT (Greece); NKFIA (Hungary); DAE and DST (India); IPM (Iran); SFI (Ireland); INFN (Italy); MSIP and NRF (Republic of Korea); MES (Latvia); LAS (Lithuania); MOE and UM (Malaysia); BUAP, CINVESTAV, CONACYT, LNS, SEP, and UASLP-FAI (Mexico); MOS (Montenegro); MBIE (New Zealand); PAEC (Pakistan); MSHE and NSC (Poland); FCT (Portugal); JINR (Dubna); MON, RosAtom, RAS, RFBR, and NRC KI (Russia); MESTD (Serbia); SEIDI, CPAN, PCTI, and FEDER (Spain); MOSTR (Sri Lanka); Swiss Funding Agencies (Switzerland); MST (Taipei); ThEPCenter, IPST, STAR, and NSTDA (Thailand); TUBITAK and TAEK (Turkey); NASU (Ukraine); STFC (United Kingdom); DOE and NSF (U.S.A.).

Individuals have received support from the Marie-Curie program and the European Research Council and Horizon 2020 Grant, contract Nos. 675440, 752730, and 765710 (European Union); the Leventis Foundation; the A.P. Sloan Foundation; the Alexander von Humboldt Foundation; the Belgian Federal Science Policy Office; the Fonds pour la Formation à la Recherche dans l'Industrie et dans l'Agriculture (FRIA-Belgium); the Agentschap voor Innovatie door Wetenschap en Technologie (IWT-Belgium); the F.R.S.-FNRS and FWO (Belgium) under the "Excellence of Science - EOS" — be.h project n. 30820817; the Beijing Municipal Science \& Technology Commission, No. Z181100004218003; the Ministry of Education, Youth and Sports (MEYS) of the Czech Republic; the Lendület ("Momentum") Program and the János Bolyai Research Scholarship of the Hungarian Academy of Sciences, the New National Excellence Program ÚNKP, the NKFIA research grants 123842, 123959, 124845, 124850, 125105, 128713, 128786, and 129058 (Hungary); the Council of Science and Industrial Research, India; the HOMING PLUS program of the Foundation for Polish Science, cofinanced from European Union, Regional Development Fund, the Mobility Plus program of the Ministry of Science and Higher Education, the National Science Center (Poland), contracts Harmonia 2014/14/M/ST2/00428, Opus 2014/13/B/ST2/02543, 2014/15/B/ST2/03998, and 2015/19/B/ST2/02861, Sonatabis 2012/07/E/ST2/01406; the National Priorities Research Program by Qatar National Research Fund; the Ministry of Science and Education, grant no. 3.2989.2017 (Russia); the Programa Estatal de Fomento de la Investigación Científica y Técnica de Excelencia María 
de Maeztu, grant MDM-2015-0509 and the Programa Severo Ochoa del Principado de Asturias; the Thalis and Aristeia programs cofinanced by EU-ESF and the Greek NSRF; the Rachadapisek Sompot Fund for Postdoctoral Fellowship, Chulalongkorn University and the Chulalongkorn Academic into Its 2nd Century Project Advancement Project (Thailand); the Nvidia Corporation; the Welch Foundation, contract C-1845; and the Weston Havens Foundation (U.S.A.).

Open Access. This article is distributed under the terms of the Creative Commons Attribution License (CC-BY 4.0), which permits any use, distribution and reproduction in any medium, provided the original author(s) and source are credited.

\section{References}

[1] L.A. Anchordoqui, H. Goldberg, D. Lüst, S. Nawata, S. Stieberger and T.R. Taylor, Dijet signals for low mass strings at the LHC, Phys. Rev. Lett. 101 (2008) 241803 [arXiv: 0808.0497] [INSPIRE].

[2] S. Cullen, M. Perelstein and M.E. Peskin, TeV strings and collider probes of large extra dimensions, Phys. Rev. D 62 (2000) 055012 [hep-ph/0001166] [INSPIRE].

[3] J.L. Hewett and T.G. Rizzo, Low-energy phenomenology of superstring inspired $E_{6}$ models, Phys. Rept. 183 (1989) 193 [INSPIRE].

[4] U. Baur, I. Hinchliffe and D. Zeppenfeld, Excited quark production at hadron colliders, Int. J. Mod. Phys. A 2 (1987) 1285 [inSPIRE].

[5] U. Baur, M. Spira and P.M. Zerwas, Excited quark and lepton production at hadron colliders, Phys. Rev. D 42 (1990) 815 [InSPIRE].

[6] P.H. Frampton and S.L. Glashow, Chiral color: an alternative to the Standard Model, Phys. Lett. B 190 (1987) 157 [INSPIRE].

[7] R.S. Chivukula, A. Farzinnia, J. Ren and E.H. Simmons, Hadron collider production of massive color-octet vector bosons at next-to-leading order, Phys. Rev. D 87 (2013) 094011 [arXiv: 1303.1120] [INSPIRE].

[8] E.H. Simmons, Coloron phenomenology, Phys. Rev. D 55 (1997) 1678 [hep-ph/9608269] [INSPIRE].

[9] T. Han, I. Lewis and Z. Liu, Colored resonant signals at the LHC: largest rate and simplest topology, JHEP 12 (2010) 085 [arXiv:1010.4309] [INSPIRE].

[10] E. Eichten, I. Hinchliffe, K.D. Lane and C. Quigg, Super collider physics, Rev. Mod. Phys. 56 (1984) 579 [INSPIRE].

[11] L. Randall and R. Sundrum, An alternative to compactification, Phys. Rev. Lett. 83 (1999) 4690 [hep-th/9906064] [INSPIRE].

[12] M. Chala, F. Kahlhoefer, M. McCullough, G. Nardini and K. Schmidt-Hoberg, Constraining dark sectors with monojets and dijets, JHEP 07 (2015) 089 [arXiv: 1503. 05916] [INSPIRE].

[13] D. Abercrombie et al., Dark matter benchmark models for early LHC run-2 searches: report of the ATLAS/CMS dark matter forum, Phys. Dark Univ. 27 (2020) 100371 [arXiv: 1507.00966] [INSPIRE]. 
[14] J. Abdallah et al., Simplified models for dark matter searches at the LHC, Phys. Dark Univ. 9-10 (2015) 8 [arXiv:1506.03116] [INSPIRE].

[15] G. Busoni et al., Recommendations on presenting LHC searches for missing transverse energy signals using simplified s-channel models of dark matter, Phys. Dark Univ. 27 (2020) 100365 [arXiv: 1603.04156] [INSPIRE].

[16] ATLAS collaboration, Search for new resonances in mass distributions of jet pairs using $139 \mathrm{fb}^{-1}$ of pp collisions at $\sqrt{s}=13 \mathrm{TeV}$ with the ATLAS detector, JHEP 03 (2020) 145 [arXiv: 1910.08447] [INSPIRE].

[17] CMS collaboration, Search for narrow and broad dijet resonances in proton-proton collisions at $\sqrt{s}=13 \mathrm{TeV}$ and constraints on dark matter mediators and other new particles, JHEP 08 (2018) 130 [arXiv: 1806.00843] [INSPIRE].

[18] ATLAS collaboration, Search for new phenomena in dijet events using $37 \mathrm{fb}^{-1}$ of $\mathrm{pp}$ collision data collected at $\sqrt{s}=13 \mathrm{TeV}$ with the ATLAS detector, Phys. Rev. D 96 (2017) 052004 [arXiv: 1703.09127] [INSPIRE].

[19] CMS collaboration, Search for dijet resonances in proton-proton collisions at $\sqrt{s}=13 \mathrm{TeV}$ and constraints on dark matter and other models, Phys. Lett. B 769 (2017) 520 [Erratum ibid. B 772 (2017) 882] [arXiv: 1611.03568] [INSPIRE].

[20] CMS collaboration, Search for narrow resonances decaying to dijets in proton-proton collisions at $\sqrt{s}=13$ TeV, Phys. Rev. Lett. 116 (2016) 071801 [arXiv:1512.01224] [INSPIRE].

[21] ATLAS collaboration, Search for new phenomena in dijet mass and angular distributions from pp collisions at $\sqrt{s}=13 \mathrm{TeV}$ with the ATLAS detector, Phys. Lett. B 754 (2016) 302 [arXiv: 1512.01530] [INSPIRE].

[22] CMS collaboration, Search for narrow resonances in dijet final states at $\sqrt{s}=8 \mathrm{TeV}$ with the novel CMS technique of data scouting, Phys. Rev. Lett. 117 (2016) 031802 [arXiv: 1604.08907] [INSPIRE].

[23] CMS collaboration, Search for resonances and quantum black holes using dijet mass spectra in proton-proton collisions at $\sqrt{s}=8 \mathrm{TeV}$, Phys. Rev. D 91 (2015) 052009 [arXiv: 1501.04198] [INSPIRE].

[24] ATLAS collaboration, Search for new phenomena in the dijet mass distribution using pp collision data at $\sqrt{s}=8 \mathrm{TeV}$ with the ATLAS detector, Phys. Rev. D 91 (2015) 052007 [arXiv:1407.1376] [INSPIRE].

[25] CMS collaboration, Search for narrow resonances using the dijet mass spectrum in pp collisions at $\sqrt{s}=8 \mathrm{TeV}$, Phys. Rev. D 87 (2013) 114015 [arXiv:1302.4794] [INSPIRE].

[26] CMS collaboration, Search for narrow resonances and quantum black holes in inclusive and $b$-tagged dijet mass spectra from pp collisions at $\sqrt{s}=7 \mathrm{TeV}$, JHEP 01 (2013) 013 [arXiv: 1210.2387] [INSPIRE].

[27] ATLAS collaboration, Search for new physics in the dijet mass distribution using $1 \mathrm{fb}^{-1}$ of pp collision data at $\sqrt{s}=7 \mathrm{TeV}$ collected by the ATLAS detector, Phys. Lett. B 708 (2012) 37 [arXiv: 1108.6311] [INSPIRE].

[28] ATLAS collaboration, ATLAS search for new phenomena in dijet mass and angular distributions using pp collisions at $\sqrt{s}=7 \mathrm{TeV}$, JHEP 01 (2013) 029 [arXiv:1210.1718] [INSPIRE]. 
[29] CMS collaboration, Search for resonances in the dijet mass spectrum from $7 \mathrm{TeV} \mathrm{pp}$ collisions at CMS, Phys. Lett. B 704 (2011) 123 [arXiv:1107.4771] [InSPIRE].

[30] ATLAS collaboration, Search for new physics in dijet mass and angular distributions in pp collisions at $\sqrt{s}=7 \mathrm{TeV}$ measured with the ATLAS detector, New J. Phys. 13 (2011) 053044 [arXiv:1103.3864] [INSPIRE].

[31] CMS collaboration, Search for dijet resonances in $7 \mathrm{TeV}$ pp collisions at CMS, Phys. Rev. Lett. 105 (2010) 211801 [Erratum ibid. 106 (2011) 029902] [arXiv:1010.0203] [INSPIRE].

[32] ATLAS collaboration, Search for new particles in two-jet final states in $7 \mathrm{TeV}$ proton-proton collisions with the ATLAS detector at the LHC, Phys. Rev. Lett. 105 (2010) 161801 [arXiv: 1008.2461] [INSPIRE].

[33] R.M. Harris and K. Kousouris, Searches for dijet resonances at hadron colliders, Int. J. Mod. Phys. A 26 (2011) 5005 [arXiv:1110.5302] [INSPIRE].

[34] R. Sekhar Chivukula, E.H. Simmons and N. Vignaroli, Distinguishing dijet resonances at the LHC, Phys. Rev. D 91 (2015) 055019 [arXiv:1412.3094] [INSPIRE].

[35] CMS collaboration, The CMS experiment at the CERN LHC, 2008 JINST 3 S08004 [INSPIRE].

[36] CMS collaboration, Particle-flow reconstruction and global event description with the CMS detector, 2017 JINST 12 P10003 [arXiv:1706.04965] [INSPIRE].

[37] M. Cacciari and G.P. Salam, Dispelling the $N^{3}$ myth for the $k_{t}$ jet-finder, Phys. Lett. B 641 (2006) 57 [hep-ph/0512210] [INSPIRE].

[38] M. Cacciari, G.P. Salam and G. Soyez, The anti- $k_{t}$ jet clustering algorithm, JHEP 04 (2008) 063 [arXiv: 0802.1189] [INSPIRE].

[39] M. Cacciari, G.P. Salam and G. Soyez, FastJet user manual, Eur. Phys. J. C 72 (2012) 1896 [arXiv: 1111.6097] [INSPIRE].

[40] M. Cacciari and G.P. Salam, Pileup subtraction using jet areas, Phys. Lett. B 659 (2008) 119 [arXiv:0707.1378] [INSPIRE].

[41] CMS collaboration, Jet energy scale and resolution in the CMS experiment in pp collisions at 8 TeV, 2017 JINST 12 P02014 [arXiv:1607.03663] [INSPIRE].

[42] CMS collaboration, The CMS trigger system, 2017 JINST 12 P01020 [arXiv:1609.02366] [INSPIRE].

[43] CMS collaboration, Jet algorithms performance in 13 TeV data, CMS-PAS-JME-16-003, CERN, Geneva, Switzerland (2017).

[44] T. Sjöstrand et al., An introduction to PYTHIA 8.2, Comput. Phys. Commun. 191 (2015) 159 [arXiv: 1410.3012] [INSPIRE].

[45] CMS collaboration, Event generator tunes obtained from underlying event and multiparton scattering measurements, Eur. Phys. J. C 76 (2016) 155 [arXiv:1512.00815] [InSPIRE].

[46] P. Skands, S. Carrazza and J. Rojo, Tuning PYTHIA 8.1: the Monash 2013 tune, Eur. Phys. J. C 74 (2014) 3024 [arXiv: 1404.5630] [InSPIRE].

[47] R.D. Ball et al., Parton distributions with LHC data, Nucl. Phys. B 867 (2013) 244 [arXiv: 1207.1303] [INSPIRE]. 
[48] GEANT4 collaboration, GEANT4: a simulation toolkit, Nucl. Instrum. Meth. A 506 (2003) 250 [INSPIRE].

[49] B.A. Dobrescu, R.M. Harris and J. Isaacson, Ultraheavy resonances at the LHC: beyond the QCD background, arXiv:1810.09429 [INSPIRE].

[50] CDF collaboration, Search for new particles decaying into dijets in proton-antiproton collisions at $\sqrt{s}=1.96 \mathrm{TeV}$, Phys. Rev. D 79 (2009) 112002 [arXiv:0812.4036] [InSPIRE].

[51] P. Nason, A new method for combining NLO QCD with shower Monte Carlo algorithms, JHEP 11 (2004) 040 [hep-ph/0409146] [INSPIRE].

[52] S. Frixione, P. Nason and C. Oleari, Matching NLO QCD computations with parton shower simulations: the POWHEG method, JHEP 11 (2007) 070 [arXiv: 0709.2092] [INSPIRE].

[53] S. Alioli, P. Nason, C. Oleari and E. Re, A general framework for implementing NLO calculations in shower Monte Carlo programs: the POWHEG BOX, JHEP 06 (2010) 043 [arXiv: 1002.2581] [INSPIRE].

[54] S. Dittmaier, A. Huss and C. Speckner, Weak radiative corrections to dijet production at hadron colliders, JHEP 11 (2012) 095 [arXiv: 1210. 0438] [INSPIRE].

[55] CMS collaboration, CMS luminosity measurements for the 2016 data taking period, CMS-PAS-LUM-17-001, CERN, Geneva, Switzerland (2017).

[56] CMS collaboration, CMS luminosity measurement for the 2018 data-taking period at $\sqrt{s}=13 \mathrm{TeV}$, CMS-PAS-LUM-18-002, CERN, Geneva, Switzerland (2018).

[57] CMS collaboration, CMS luminosity measurement for the 2017 data-taking period at $\sqrt{s}=13 \mathrm{TeV}$, CMS-PAS-LUM-17-004, CERN, Geneva, Switzerland (2017).

[58] T. Junk, Confidence level computation for combining searches with small statistics, Nucl. Instrum. Meth. A 434 (1999) 435 [hep-ex/9902006] [INSPIRE].

[59] A.L. Read, Presentation of search results: the $C L_{s}$ technique, J. Phys. G 28 (2002) 2693 [INSPIRE].

[60] ATLAS, CMS collaborations and the LHC Higgs Combination Group, Procedure for the LHC Higgs boson search combination in Summer 2011, CMS-NOTE-2011-005, CERN, Geneva, Switzerland (2011).

[61] G. Cowan, K. Cranmer, E. Gross and O. Vitells, Asymptotic formulae for likelihood-based tests of new physics, Eur. Phys. J. C 71 (2011) 1554 [Erratum ibid. C 73 (2013) 2501] [arXiv: 1007.1727] [INSPIRE].

[62] J. Pumplin, D.R. Stump, J. Huston, H.L. Lai, P.M. Nadolsky and W.K. Tung, New generation of parton distributions with uncertainties from global QCD analysis, JHEP 07 (2002) 012 [hep-ph/0201195] [INSPIRE].

[63] V.D. Barger and R.J.N. Phillips, Collider physics, updated edition, Front. Phys. 71, Westview Press, Boulder, CO, U.S.A. (1996) [ISBN:0-201-14945-1].

[64] J. Alwall et al., The automated computation of tree-level and next-to-leading order differential cross sections and their matching to parton shower simulations, JHEP 07 (2014) 079 [arXiv: 1405.0301] [INSPIRE].

[65] B.A. Dobrescu and F. Yu, Coupling-mass mapping of dijet peak searches, Phys. Rev. D 88 (2013) 035021 [Erratum ibid. D 90 (2014) 079901] [arXiv: 1306.2629] [InSPIRE]. 


\section{The CMS collaboration}

\section{Yerevan Physics Institute, Yerevan, Armenia}

A.M. Sirunyan ${ }^{\dagger}$, A. Tumasyan

\section{Institut für Hochenergiephysik, Wien, Austria}

W. Adam, F. Ambrogi, T. Bergauer, M. Dragicevic, J. Erö, A. Escalante Del Valle, M. Flechl, R. Frühwirth ${ }^{1}$, M. Jeitler ${ }^{1}$, N. Krammer, I. Krätschmer, D. Liko, T. Madlener, I. Mikulec, N. Rad, J. Schieck ${ }^{1}$, R. Schöfbeck, M. Spanring, D. Spitzbart, W. Waltenberger, C.-E. Wulz ${ }^{1}$, M. Zarucki

Institute for Nuclear Problems, Minsk, Belarus

V. Drugakov, V. Mossolov, J. Suarez Gonzalez

Universiteit Antwerpen, Antwerpen, Belgium

M.R. Darwish, E.A. De Wolf, D. Di Croce, X. Janssen, A. Lelek, M. Pieters, H. Rejeb Sfar, H. Van Haevermaet, P. Van Mechelen, S. Van Putte, N. Van Remortel

\section{Vrije Universiteit Brussel, Brussel, Belgium}

F. Blekman, E.S. Bols, S.S. Chhibra, J. D'Hondt, J. De Clercq, D. Lontkovskyi, S. Lowette, I. Marchesini, S. Moortgat, Q. Python, K. Skovpen, S. Tavernier, W. Van Doninck, P. Van Mulders

\section{Université Libre de Bruxelles, Bruxelles, Belgium}

D. Beghin, B. Bilin, B. Clerbaux, G. De Lentdecker, H. Delannoy, B. Dorney, L. Favart, A. Grebenyuk, A.K. Kalsi, A. Popov, N. Postiau, E. Starling, L. Thomas, C. Vander Velde, P. Vanlaer, D. Vannerom

\section{Ghent University, Ghent, Belgium}

T. Cornelis, D. Dobur, I. Khvastunov², M. Niedziela, C. Roskas, M. Tytgat, W. Verbeke, B. Vermassen, M. Vit

\section{Université Catholique de Louvain, Louvain-la-Neuve, Belgium}

O. Bondu, G. Bruno, C. Caputo, P. David, C. Delaere, M. Delcourt, A. Giammanco, V. Lemaitre, J. Prisciandaro, A. Saggio, M. Vidal Marono, P. Vischia, J. Zobec

\section{Centro Brasileiro de Pesquisas Fisicas, Rio de Janeiro, Brazil}

F.L. Alves, G.A. Alves, G. Correia Silva, C. Hensel, A. Moraes, P. Rebello Teles

Universidade do Estado do Rio de Janeiro, Rio de Janeiro, Brazil

E. Belchior Batista Das Chagas, W. Carvalho, J. Chinellato ${ }^{3}$, E. Coelho, E.M. Da Costa, G.G. Da Silveira ${ }^{4}$, D. De Jesus Damiao, C. De Oliveira Martins, S. Fonseca De Souza, L.M. Huertas Guativa, H. Malbouisson, J. Martins ${ }^{5}$, D. Matos Figueiredo, M. Medina Jaime ${ }^{6}$, M. Melo De Almeida, C. Mora Herrera, L. Mundim, H. Nogima, W.L. Prado Da Silva, L.J. Sanchez Rosas, A. Santoro, A. Sznajder, M. Thiel, E.J. Tonelli Manganote ${ }^{3}$, F. Torres Da Silva De Araujo, A. Vilela Pereira 
Universidade Estadual Paulista ${ }^{a}$, Universidade Federal do $\mathrm{ABC}^{b}$, São Paulo, Brazil

C.A. Bernardes ${ }^{a}$, L. Calligaris ${ }^{a}$, T.R. Fernandez Perez Tomei ${ }^{a}$, E.M. Gregores ${ }^{b}$, D.S. Lemos, P.G. Mercadante ${ }^{b}$, S.F. Novaes ${ }^{a}$, SandraS. Padula ${ }^{a}$

Institute for Nuclear Research and Nuclear Energy, Bulgarian Academy of Sciences, Sofia, Bulgaria

A. Aleksandrov, G. Antchev, R. Hadjiiska, P. Iaydjiev, M. Misheva, M. Rodozov, M. Shopova, G. Sultanov

University of Sofia, Sofia, Bulgaria

M. Bonchev, A. Dimitrov, T. Ivanov, L. Litov, B. Pavlov, P. Petkov

Beihang University, Beijing, China

W. Fang $^{7}$, X. Gao ${ }^{7}$, L. Yuan

Department of Physics, Tsinghua University, Beijing, China

M. Ahmad, Z. Hu, Y. Wang

Institute of High Energy Physics, Beijing, China

G.M. Chen, H.S. Chen, M. Chen, C.H. Jiang, D. Leggat, H. Liao, Z. Liu, A. Spiezia, J. Tao, E. Yazgan, H. Zhang, S. Zhang ${ }^{8}$, J. Zhao

State Key Laboratory of Nuclear Physics and Technology, Peking University, Beijing, China

A. Agapitos, Y. Ban, G. Chen, A. Levin, J. Li, L. Li, Q. Li, Y. Mao, S.J. Qian, D. Wang, Q. Wang

Zhejiang University, Hangzhou, China

M. Xiao

Universidad de Los Andes, Bogota, Colombia

C. Avila, A. Cabrera, C. Florez, C.F. González Hernández, M.A. Segura Delgado

Universidad de Antioquia, Medellin, Colombia

J. Mejia Guisao, J.D. Ruiz Alvarez, C.A. Salazar González, N. Vanegas Arbelaez

University of Split, Faculty of Electrical Engineering, Mechanical Engineering and Naval Architecture, Split, Croatia

D. Giljanović, N. Godinovic, D. Lelas, I. Puljak, T. Sculac

University of Split, Faculty of Science, Split, Croatia

Z. Antunovic, M. Kovac

Institute Rudjer Boskovic, Zagreb, Croatia

V. Brigljevic, D. Ferencek, K. Kadija, B. Mesic, M. Roguljic, A. Starodumov ${ }^{9}$, T. Susa

University of Cyprus, Nicosia, Cyprus

M.W. Ather, A. Attikis, E. Erodotou, A. Ioannou, M. Kolosova, S. Konstantinou,

G. Mavromanolakis, J. Mousa, C. Nicolaou, F. Ptochos, P.A. Razis, H. Rykaczewski,

D. Tsiakkouri 
Charles University, Prague, Czech Republic

M. Finger ${ }^{10}$, M. Finger Jr. ${ }^{10}$, A. Kveton, J. Tomsa

Escuela Politecnica Nacional, Quito, Ecuador

E. Ayala

Universidad San Francisco de Quito, Quito, Ecuador

E. Carrera Jarrin

Academy of Scientific Research and Technology of the Arab Republic of Egypt, Egyptian Network of High Energy Physics, Cairo, Egypt

Y. Assran ${ }^{11,12}$, S. Elgammal ${ }^{12}$

National Institute of Chemical Physics and Biophysics, Tallinn, Estonia

S. Bhowmik, A. Carvalho Antunes De Oliveira, R.K. Dewanjee, K. Ehataht, M. Kadastik, M. Raidal, C. Veelken

Department of Physics, University of Helsinki, Helsinki, Finland

P. Eerola, L. Forthomme, H. Kirschenmann, K. Osterberg, M. Voutilainen

Helsinki Institute of Physics, Helsinki, Finland

F. Garcia, J. Havukainen, J.K. Heikkilä, V. Karimäki, M.S. Kim, R. Kinnunen, T. Lampén,

K. Lassila-Perini, S. Laurila, S. Lehti, T. Lindén, P. Luukka, T. Mäenpää, H. Siikonen,

E. Tuominen, J. Tuominiemi

Lappeenranta University of Technology, Lappeenranta, Finland

T. Tuuva

IRFU, CEA, Université Paris-Saclay, Gif-sur-Yvette, France

M. Besancon, F. Couderc, M. Dejardin, D. Denegri, B. Fabbro, J.L. Faure, F. Ferri,

S. Ganjour, A. Givernaud, P. Gras, G. Hamel de Monchenault, P. Jarry, C. Leloup,

B. Lenzi, E. Locci, J. Malcles, J. Rander, A. Rosowsky, M.Ö. Sahin, A. Savoy-Navarro ${ }^{13}$,

M. Titov, G.B. Yu

Laboratoire Leprince-Ringuet, CNRS/IN2P3, Ecole Polytechnique, Institut Polytechnique de Paris

S. Ahuja, C. Amendola, F. Beaudette, P. Busson, C. Charlot, B. Diab, G. Falmagne,

R. Granier de Cassagnac, I. Kucher, A. Lobanov, C. Martin Perez, M. Nguyen, C. Ochando,

P. Paganini, J. Rembser, R. Salerno, J.B. Sauvan, Y. Sirois, A. Zabi, A. Zghiche

Université de Strasbourg, CNRS, IPHC UMR 7178, Strasbourg, France

J.-L. Agram ${ }^{14}$, J. Andrea, D. Bloch, G. Bourgatte, J.-M. Brom, E.C. Chabert, C. Collard,

E. Conte ${ }^{14}$, J.-C. Fontaine ${ }^{14}$, D. Gelé, U. Goerlach, M. Jansová, A.-C. Le Bihan, N. Tonon,

P. Van Hove

Centre de Calcul de l'Institut National de Physique Nucleaire et de Physique des Particules, CNRS/IN2P3, Villeurbanne, France

S. Gadrat 
Université de Lyon, Université Claude Bernard Lyon 1, CNRS-IN2P3, Institut de Physique Nucléaire de Lyon, Villeurbanne, France

S. Beauceron, C. Bernet, G. Boudoul, C. Camen, A. Carle, N. Chanon, R. Chierici, D. Contardo, P. Depasse, H. El Mamouni, J. Fay, S. Gascon, M. Gouzevitch, B. Ille, Sa. Jain, F. Lagarde, I.B. Laktineh, H. Lattaud, A. Lesauvage, M. Lethuillier, L. Mirabito, S. Perries, V. Sordini, L. Torterotot, G. Touquet, M. Vander Donckt, S. Viret

Georgian Technical University, Tbilisi, Georgia

T. Toriashvili ${ }^{15}$

Tbilisi State University, Tbilisi, Georgia

Z. Tsamalaidze ${ }^{10}$

RWTH Aachen University, I. Physikalisches Institut, Aachen, Germany

C. Autermann, L. Feld, K. Klein, M. Lipinski, D. Meuser, A. Pauls, M. Preuten, M.P. Rauch, J. Schulz, M. Teroerde, B. Wittmer

RWTH Aachen University, III. Physikalisches Institut A, Aachen, Germany

M. Erdmann, B. Fischer, S. Ghosh, T. Hebbeker, K. Hoepfner, H. Keller, L. Mastrolorenzo, M. Merschmeyer, A. Meyer, P. Millet, G. Mocellin, S. Mondal, S. Mukherjee, D. Noll, A. Novak, T. Pook, A. Pozdnyakov, T. Quast, M. Radziej, Y. Rath, H. Reithler, J. Roemer, A. Schmidt, S.C. Schuler, A. Sharma, S. Wiedenbeck, S. Zaleski

RWTH Aachen University, III. Physikalisches Institut B, Aachen, Germany G. Flügge, W. Haj Ahmad ${ }^{16}$, O. Hlushchenko, T. Kress, T. Müller, A. Nowack, C. Pistone, O. Pooth, D. Roy, H. Sert, A. Stahl ${ }^{17}$

\section{Deutsches Elektronen-Synchrotron, Hamburg, Germany}

M. Aldaya Martin, P. Asmuss, I. Babounikau, H. Bakhshiansohi, K. Beernaert, O. Behnke, A. Bermúdez Martínez, D. Bertsche, A.A. Bin Anuar, K. Borras ${ }^{18}$, V. Botta, A. Campbell, A. Cardini, P. Connor, S. Consuegra Rodríguez, C. Contreras-Campana, V. Danilov, A. De Wit, M.M. Defranchis, C. Diez Pardos, D. Domínguez Damiani, G. Eckerlin, D. Eckstein, T. Eichhorn, A. Elwood, E. Eren, E. Gallo ${ }^{19}$, A. Geiser, A. Grohsjean, M. Guthoff, M. Haranko, A. Harb, A. Jafari, N.Z. Jomhari, H. Jung, A. Kasem ${ }^{18}$, M. Kasemann, H. Kaveh, J. Keaveney, C. Kleinwort, J. Knolle, D. Krücker, W. Lange, T. Lenz, J. Lidrych, K. Lipka, W. Lohmann ${ }^{20}$, R. Mankel, I.-A. Melzer-Pellmann, A.B. Meyer, M. Meyer, M. Missiroli, J. Mnich, A. Mussgiller, V. Myronenko, D. Pérez Adán, S.K. Pflitsch, D. Pitzl, A. Raspereza, A. Saibel, M. Savitskyi, V. Scheurer, P. Schütze, C. Schwanenberger, R. Shevchenko, A. Singh, H. Tholen, O. Turkot, A. Vagnerini, M. Van De Klundert, R. Walsh, Y. Wen, K. Wichmann, C. Wissing, O. Zenaiev, R. Zlebcik

\section{University of Hamburg, Hamburg, Germany}

R. Aggleton, S. Bein, L. Benato, A. Benecke, V. Blobel, T. Dreyer, A. Ebrahimi, F. Feindt, A. Fröhlich, C. Garbers, E. Garutti, D. Gonzalez, P. Gunnellini, J. Haller, A. Hinzmann, A. Karavdina, G. Kasieczka, R. Klanner, R. Kogler, N. Kovalchuk, S. Kurz, V. Kutzner, J. Lange, T. Lange, A. Malara, J. Multhaup, C.E.N. Niemeyer, A. Perieanu, A. Reimers, 
O. Rieger, C. Scharf, P. Schleper, S. Schumann, J. Schwandt, J. Sonneveld, H. Stadie, G. Steinbrück, F.M. Stober, B. Vormwald, I. Zoi

Karlsruher Institut fuer Technologie, Karlsruhe, Germany

M. Akbiyik, C. Barth, M. Baselga, S. Baur, T. Berger, E. Butz, R. Caspart, T. Chwalek,

W. De Boer, A. Dierlamm, K. El Morabit, N. Faltermann, M. Giffels, P. Goldenzweig, A. Gottmann, M.A. Harrendorf, F. Hartmann ${ }^{17}$, U. Husemann, S. Kudella, S. Mitra, M.U. Mozer, D. Müller, Th. Müller, M. Musich, A. Nürnberg, G. Quast, K. Rabbertz, M. Schröder, I. Shvetsov, H.J. Simonis, R. Ulrich, M. Wassmer, M. Weber, C. Wöhrmann, R. Wolf

Institute of Nuclear and Particle Physics (INPP), NCSR Demokritos, Aghia Paraskevi, Greece

G. Anagnostou, P. Asenov, G. Daskalakis, T. Geralis, A. Kyriakis, D. Loukas, G. Paspalaki

National and Kapodistrian University of Athens, Athens, Greece

M. Diamantopoulou, D. Karasavvas, G. Karathanasis, P. Kontaxakis, A. Manousakiskatsikakis, A. Panagiotou, I. Papavergou, N. Saoulidou, A. Stakia, K. Theofilatos, K. Vellidis, E. Vourliotis

National Technical University of Athens, Athens, Greece

G. Bakas, K. Kousouris, I. Papakrivopoulos, G. Tsipolitis

University of Ioánnina, Ioánnina, Greece

I. Evangelou, C. Foudas, P. Gianneios, P. Katsoulis, P. Kokkas, S. Mallios, K. Manitara, N. Manthos, I. Papadopoulos, J. Strologas, F.A. Triantis, D. Tsitsonis

MTA-ELTE Lendület CMS Particle and Nuclear Physics Group, Eötvös Loránd University, Budapest, Hungary

M. Bartók ${ }^{21}$, R. Chudasama, M. Csanad, P. Major, K. Mandal, A. Mehta, M.I. Nagy, G. Pasztor, O. Surányi, G.I. Veres

Wigner Research Centre for Physics, Budapest, Hungary

G. Bencze, C. Hajdu, D. Horvath ${ }^{22}$, F. Sikler, T.Á. Vámi, V. Veszpremi, G. Vesztergombi ${ }^{\dagger}$

Institute of Nuclear Research ATOMKI, Debrecen, Hungary

N. Beni, S. Czellar, J. Karancsi ${ }^{21}$, J. Molnar, Z. Szillasi

Institute of Physics, University of Debrecen, Debrecen, Hungary

P. Raics, D. Teyssier, Z.L. Trocsanyi, B. Ujvari

Eszterhazy Karoly University, Karoly Robert Campus, Gyongyos, Hungary

T. Csorgo, W.J. Metzger, F. Nemes, T. Novak

Indian Institute of Science (IISc), Bangalore, India

S. Choudhury, J.R. Komaragiri, P.C. Tiwari 
National Institute of Science Education and Research, HBNI, Bhubaneswar, India

S. Bahinipati ${ }^{24}$, C. Kar, G. Kole, P. Mal, V.K. Muraleedharan Nair Bindhu, A. Nayak ${ }^{25}$, D.K. Sahoo ${ }^{24}$, S.K. Swain

Panjab University, Chandigarh, India

S. Bansal, S.B. Beri, V. Bhatnagar, S. Chauhan, R. Chawla, N. Dhingra, R. Gupta, A. Kaur, M. Kaur, S. Kaur, P. Kumari, M. Lohan, M. Meena, K. Sandeep, S. Sharma, J.B. Singh, A.K. Virdi, G. Walia

University of Delhi, Delhi, India

A. Bhardwaj, B.C. Choudhary, R.B. Garg, M. Gola, S. Keshri, Ashok Kumar, M. Naimuddin, P. Priyanka, K. Ranjan, Aashaq Shah, R. Sharma

Saha Institute of Nuclear Physics, HBNI, Kolkata, India

R. Bhardwaj ${ }^{26}$, M. Bharti ${ }^{26}$, R. Bhattacharya, S. Bhattacharya, U. Bhawandeep ${ }^{26}$, D. Bhowmik, S. Dutta, S. Ghosh, B. Gomber ${ }^{27}$, M. Maity ${ }^{28}$, K. Mondal, S. Nandan, A. Purohit, P.K. Rout, G. Saha, S. Sarkar, T. Sarkar ${ }^{28}$, M. Sharan, B. Singh ${ }^{26}$, S. Thakur ${ }^{26}$

Indian Institute of Technology Madras, Madras, India

P.K. Behera, P. Kalbhor, A. Muhammad, P.R. Pujahari, A. Sharma, A.K. Sikdar

Bhabha Atomic Research Centre, Mumbai, India

D. Dutta, V. Jha, V. Kumar, D.K. Mishra, P.K. Netrakanti, L.M. Pant, P. Shukla

Tata Institute of Fundamental Research-A, Mumbai, India

T. Aziz, M.A. Bhat, S. Dugad, G.B. Mohanty, N. Sur, RavindraKumar Verma

Tata Institute of Fundamental Research-B, Mumbai, India

S. Banerjee, S. Bhattacharya, S. Chatterjee, P. Das, M. Guchait, S. Karmakar, S. Kumar, G. Majumder, K. Mazumdar, N. Sahoo, S. Sawant

Indian Institute of Science Education and Research (IISER), Pune, India

S. Dube, B. Kansal, A. Kapoor, K. Kothekar, S. Pandey, A. Rane, A. Rastogi, S. Sharma

Institute for Research in Fundamental Sciences (IPM), Tehran, Iran

S. Chenarani ${ }^{29}$, E. Eskandari Tadavani, S.M. Etesami ${ }^{29}$, M. Khakzad, M. Mohammadi Najafabadi, M. Naseri, F. Rezaei Hosseinabadi

University College Dublin, Dublin, Ireland

M. Felcini, M. Grunewald

INFN Sezione di Bari ${ }^{a}$, Università di Bari ${ }^{b}$, Politecnico di Bari ${ }^{c}$, Bari, Italy M. Abbrescia ${ }^{a, b}$, R. Aly ${ }^{a, b, 30}$, C. Calabria ${ }^{a, b}$, A. Colaleo ${ }^{a}$, D. Creanza ${ }^{a, c}$, L. Cristella $^{a, b}$, N. De Filippis ${ }^{a, c}$, M. De Palma ${ }^{a, b}$, A. Di Florio ${ }^{a, b}$, W. Elmetenawee ${ }^{a, b}$, L. Fiore ${ }^{a}$,

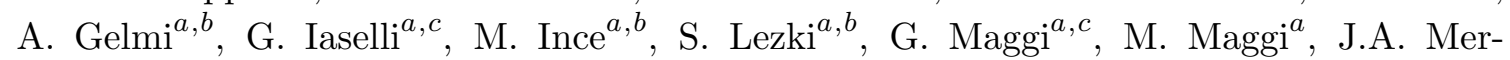
lin, G. Miniello ${ }^{a, b}$, S. My ${ }^{a, b}$, S. Nuzzo ${ }^{a, b}$, A. Pompili ${ }^{a, b}$, G. Pugliese ${ }^{a, c}$, R. Radogna ${ }^{a}$,

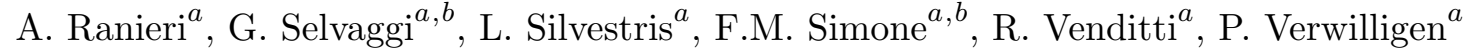


INFN Sezione di Bologna ${ }^{a}$, Università di Bologna ${ }^{b}$, Bologna, Italy

G. Abbiendi ${ }^{a}$, C. Battilana ${ }^{a, b}$, D. Bonacorsi ${ }^{a, b}$, L. Borgonovi ${ }^{a, b}$, S. Braibant-Giacomelli ${ }^{a, b}$,

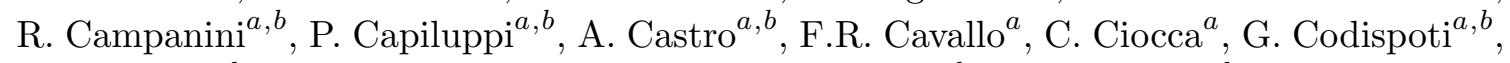
M. Cuffiani ${ }^{a, b}$, G.M. Dallavalle ${ }^{a}$, F. Fabbri ${ }^{a}$, A. Fanfani ${ }^{a, b}$, E. Fontanesi $^{a, b}$, P. Giacomelli $^{a}$, C. Grandi ${ }^{a}$, L. Guiducci ${ }^{a, b}$, F. Iemmi ${ }^{a, b}$, S. Lo Meo ${ }^{a, 31}$, S. Marcellini ${ }^{a}$, G. Masetti ${ }^{a}$,

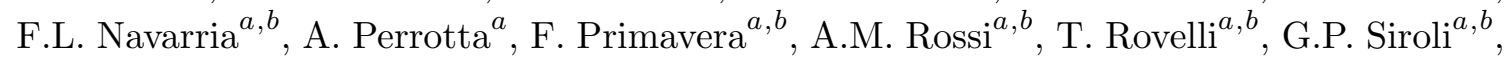
N. $\operatorname{Tosi}^{a}$

INFN Sezione di Catania $^{a}$, Università di Catania ${ }^{b}$, Catania, Italy

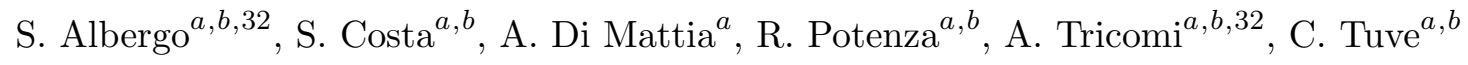

INFN Sezione di Firenze ${ }^{a}$, Università di Firenze ${ }^{b}$, Firenze, Italy

G. Barbagli ${ }^{a}$, A. Cassese, R. Ceccarelli, V. Ciulli ${ }^{a, b}$, C. Civinini $^{a}$, R. D'Alessandro ${ }^{a, b}$, F. Fiori ${ }^{a, c}$, E. Focardi ${ }^{a, b}$, G. Latino ${ }^{a, b}$, P. Lenzi ${ }^{a, b}$, M. Meschini ${ }^{a}$, S. Paoletti ${ }^{a}$, G. Sguazzoni ${ }^{a}$, L. Viliani $^{a}$

INFN Laboratori Nazionali di Frascati, Frascati, Italy

L. Benussi, S. Bianco, D. Piccolo

INFN Sezione di Genova ${ }^{a}$, Università di Genova ${ }^{b}$, Genova, Italy

M. Bozzo ${ }^{a, b}$, F. Ferro ${ }^{a}$, R. Mulargia ${ }^{a, b}$, E. Robutti $^{a}$, S. Tosi $^{a, b}$

INFN Sezione di Milano-Bicocca ${ }^{a}$, Università di Milano-Bicocca ${ }^{b}$, Milano, Italy

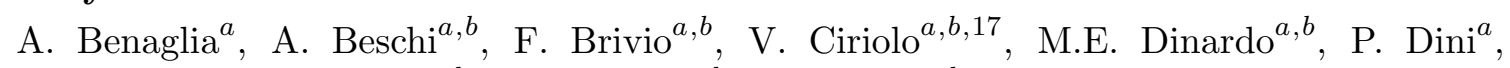
S. Gennai ${ }^{a}$, A. Ghezzi ${ }^{a, b}$, P. Govoni ${ }^{a, b}$, L. Guzzi ${ }^{a, b}$, M. Malberti ${ }^{a}$, S. Malvezzi ${ }^{a}$, D. Menasce ${ }^{a}$, F. Monti ${ }^{a, b}$, L. Moroni ${ }^{a}$, M. Paganoni ${ }^{a, b}$, D. Pedrini ${ }^{a}$, S. Ragazzi ${ }^{a, b}$, T. Tabarelli de Fatis ${ }^{a, b}$, D. Valsecchi ${ }^{a, b}$, D. Zuolo ${ }^{a, b}$

INFN Sezione di Napoli ${ }^{a}$, Università di Napoli 'Federico II' ${ }^{b}$, Napoli, Italy, Università della Basilicata $^{c}$, Potenza, Italy, Università G. Marconi ${ }^{d}$, Roma, Italy

S. Buontempo ${ }^{a}$, N. Cavallo ${ }^{a, c}$, A. De Iorio ${ }^{a, b}$, A. Di Crescenzo ${ }^{a, b}$, F. Fabozzi $^{a, c}$, F. Fienga $^{a}$, G. Galati ${ }^{a}$, A.O.M. Iorio ${ }^{a, b}$, L. Lista ${ }^{a, b}$, S. Meola ${ }^{a, d, 17}$, P. Paolucci ${ }^{a, 17}$, B. Rossi ${ }^{a}$, C. Sciacca ${ }^{a, b}$, E. Voevodina ${ }^{a, b}$

INFN Sezione di Padova ${ }^{a}$, Università di Padova ${ }^{b}$, Padova, Italy, Università di Trento ${ }^{c}$, Trento, Italy

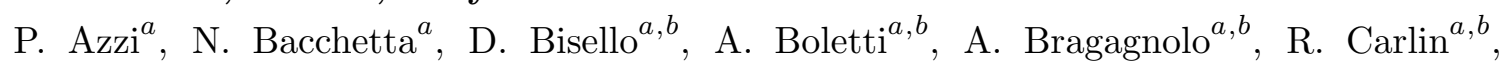

P. Checchia ${ }^{a}$, P. De Castro Manzano ${ }^{a}$, T. Dorigo ${ }^{a}$, U. Dosselli ${ }^{a}$, F. Gasparini ${ }^{a, b}$,

U. Gasparini $^{a, b}$, A. Gozzelino ${ }^{a}$, S.Y. Hoh $^{a, b}$, P. Lujan ${ }^{a}$, M. Margoni $^{a, b}$, A.T. Meneguzzo ${ }^{a, b}$,

J. Pazzini ${ }^{a, b}$, M. Presilla ${ }^{b}$, P. Ronchese ${ }^{a, b}$, R. Rossin ${ }^{a, b}$, F. Simonetto ${ }^{a, b}$, A. Tiko ${ }^{a}$, M. Tosi ${ }^{a, b}$, M. Zanetti ${ }^{a, b}$, P. Zotto ${ }^{a, b}$, G. Zumerle ${ }^{a, b}$

INFN Sezione di Pavia ${ }^{a}$, Università di Pavia ${ }^{b}$, Pavia, Italy

A. Braghieri ${ }^{a}$, D. Fiorina ${ }^{a, b}$, P. Montagna ${ }^{a, b}$, S.P. Ratti ${ }^{a, b}$, V. $\operatorname{Re}^{a}$, M. Ressegotti ${ }^{a, b}$,

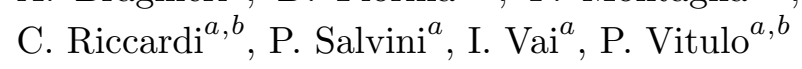


INFN Sezione di Perugia ${ }^{a}$, Università di Perugia ${ }^{b}$, Perugia, Italy

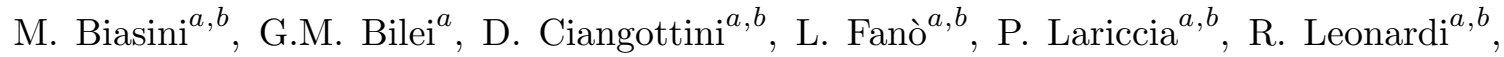

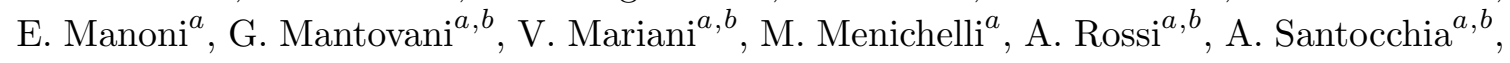

D. Spiga ${ }^{a}$

INFN Sezione di Pisa ${ }^{a}$, Università di Pisa ${ }^{b}$, Scuola Normale Superiore di Pisa c, Pisa, Italy

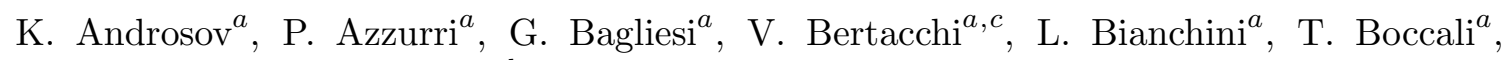
R. Castaldi $^{a}$, M.A. Ciocci ${ }^{a, b}$, R. Dell'Orso ${ }^{a}$, S. Donato ${ }^{a}$, G. Fedi ${ }^{a}$, L. Giannini ${ }^{a, c}$,

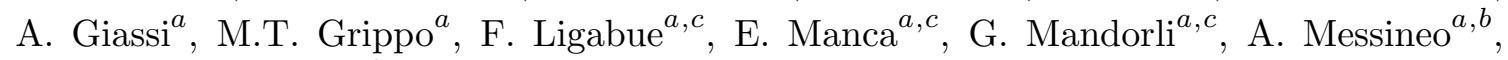
F. Palla ${ }^{a}$, A. Rizzi ${ }^{a, b}$, G. Rolandi ${ }^{33}$, S. Roy Chowdhury, A. Scribano ${ }^{a}$, P. Spagnolo ${ }^{a}$,

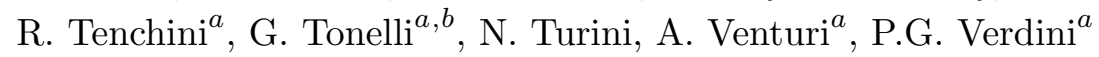

INFN Sezione di Roma ${ }^{a}$, Sapienza Università di Roma ${ }^{b}$, Rome, Italy F. Cavallari ${ }^{a}$, M. Cipriani ${ }^{a, b}$, D. Del Re ${ }^{a, b}$, E. Di Marco ${ }^{a}$, M. Diemoz $^{a}$, E. Longo ${ }^{a, b}$, P. Meridiani ${ }^{a}$, G. Organtini ${ }^{a, b}$, F. Pandolfi ${ }^{a}$, R. Paramatti ${ }^{a, b}$, C. Quaranta ${ }^{a, b}$, S. Rahatlou ${ }^{a, b}$, C. Rovelli $^{a}$, F. Santanastasio ${ }^{a, b}$, L. Soffi $^{a, b}$

INFN Sezione di Torino ${ }^{a}$, Università di Torino ${ }^{b}$, Torino, Italy, Università del Piemonte Orientale ${ }^{c}$, Novara, Italy

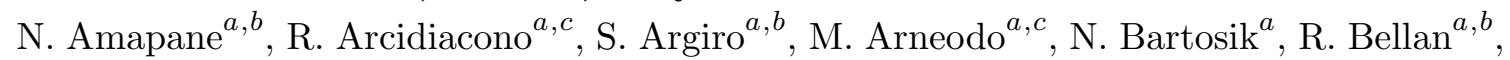
A. Bellora, C. Biino ${ }^{a}$, A. Cappati ${ }^{a, b}$, N. Cartiglia ${ }^{a}$, S. Cometti ${ }^{a}$, M. Costa $^{a, b}$, R. Covarelli $^{a, b}$, N. Demaria ${ }^{a}$, B. Kiani ${ }^{a, b}$, F. Legger, C. Mariotti ${ }^{a}$, S. Maselli ${ }^{a}$, E. Migliore $^{a, b}$, V. Monaco ${ }^{a, b}$, E. Monteil ${ }^{a, b}$, M. Monteno $^{a}$, M.M. Obertino $^{a, b}$, G. Ortona $^{a, b}$, L. Pacher ${ }^{a, b}$, N. Pastrone ${ }^{a}$, M. Pelliccioni ${ }^{a}$, G.L. Pinna Angioni ${ }^{a, b}$, A. Romero ${ }^{a, b}$,

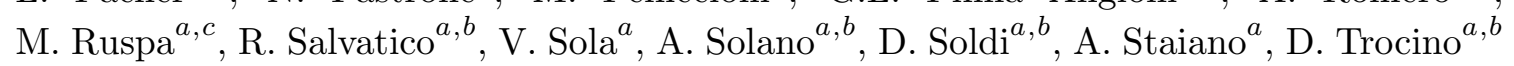

INFN Sezione di Trieste ${ }^{a}$, Università di Trieste ${ }^{b}$, Trieste, Italy

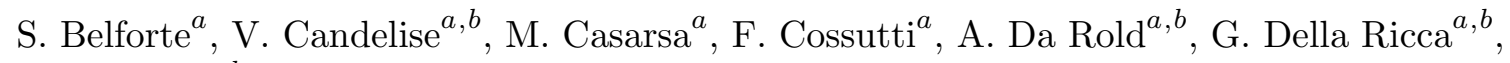
F. Vazzoler ${ }^{a, b}$, A. Zanetti ${ }^{a}$

Kyungpook National University, Daegu, Korea

B. Kim, D.H. Kim, G.N. Kim, J. Lee, S.W. Lee, C.S. Moon, Y.D. Oh, S.I. Pak, S. Sekmen, D.C. Son, Y.C. Yang

Chonnam National University, Institute for Universe and Elementary Particles, Kwangju, Korea

H. Kim, D.H. Moon, G. Oh

Hanyang University, Seoul, Korea

B. Francois, T.J. Kim, J. Park

Korea University, Seoul, Korea

S. Cho, S. Choi, Y. Go, S. Ha, B. Hong, K. Lee, K.S. Lee, J. Lim, J. Park, S.K. Park, Y. Roh, J. Yoo

Kyung Hee University, Department of Physics

J. Goh 
Sejong University, Seoul, Korea

H.S. Kim

Seoul National University, Seoul, Korea

J. Almond, J.H. Bhyun, J. Choi, S. Jeon, J. Kim, J.S. Kim, H. Lee, K. Lee, S. Lee, K. Nam, M. Oh, S.B. Oh, B.C. Radburn-Smith, U.K. Yang, H.D. Yoo, I. Yoon

University of Seoul, Seoul, Korea

D. Jeon, J.H. Kim, J.S.H. Lee, I.C. Park, I.J Watson

Sungkyunkwan University, Suwon, Korea

Y. Choi, C. Hwang, Y. Jeong, J. Lee, Y. Lee, I. Yu

Riga Technical University, Riga, Latvia

V. Veckalns ${ }^{34}$

Vilnius University, Vilnius, Lithuania

V. Dudenas, A. Juodagalvis, A. Rinkevicius, G. Tamulaitis, J. Vaitkus

National Centre for Particle Physics, Universiti Malaya, Kuala Lumpur, Malaysia

Z.A. Ibrahim, F. Mohamad Idris ${ }^{35}$, W.A.T. Wan Abdullah, M.N. Yusli, Z. Zolkapli

Universidad de Sonora (UNISON), Hermosillo, Mexico

J.F. Benitez, A. Castaneda Hernandez, J.A. Murillo Quijada, L. Valencia Palomo

Centro de Investigacion y de Estudios Avanzados del IPN, Mexico City, Mexico

H. Castilla-Valdez, E. De La Cruz-Burelo, I. Heredia-De La Cruz ${ }^{36}$, R. Lopez-Fernandez,

A. Sanchez-Hernandez

Universidad Iberoamericana, Mexico City, Mexico

S. Carrillo Moreno, C. Oropeza Barrera, M. Ramirez-Garcia, F. Vazquez Valencia

Benemerita Universidad Autonoma de Puebla, Puebla, Mexico

J. Eysermans, I. Pedraza, H.A. Salazar Ibarguen, C. Uribe Estrada

Universidad Autónoma de San Luis Potosí, San Luis Potosí, Mexico

A. Morelos Pineda

University of Montenegro, Podgorica, Montenegro

J. Mijuskovic ${ }^{2}$, N. Raicevic

University of Auckland, Auckland, New Zealand

D. Krofcheck

University of Canterbury, Christchurch, New Zealand

S. Bheesette, P.H. Butler

National Centre for Physics, Quaid-I-Azam University, Islamabad, Pakistan

A. Ahmad, M. Ahmad, Q. Hassan, H.R. Hoorani, W.A. Khan, M.A. Shah, M. Shoaib, M. Waqas 
AGH University of Science and Technology Faculty of Computer Science, Electronics and Telecommunications, Krakow, Poland

V. Avati, L. Grzanka, M. Malawski

National Centre for Nuclear Research, Swierk, Poland

H. Bialkowska, M. Bluj, B. Boimska, M. Górski, M. Kazana, M. Szleper, P. Zalewski

Institute of Experimental Physics, Faculty of Physics, University of Warsaw, Warsaw, Poland

K. Bunkowski, A. Byszuk ${ }^{37}$, K. Doroba, A. Kalinowski, M. Konecki, J. Krolikowski, M. Olszewski, M. Walczak

Laboratório de Instrumentação e Física Experimental de Partículas, Lisboa, Portugal

M. Araujo, P. Bargassa, D. Bastos, A. Di Francesco, P. Faccioli, B. Galinhas, M. Gallinaro, J. Hollar, N. Leonardo, T. Niknejad, J. Seixas, K. Shchelina, G. Strong, O. Toldaiev, J. Varela

Joint Institute for Nuclear Research, Dubna, Russia

S. Afanasiev, P. Bunin, M. Gavrilenko, I. Golutvin, I. Gorbunov, A. Kamenev, V. Karjavine, A. Lanev, A. Malakhov, V. Matveev ${ }^{38,39}$, P. Moisenz, V. Palichik, V. Perelygin, M. Savina, S. Shmatov, S. Shulha, N. Skatchkov, V. Smirnov, N. Voytishin, A. Zarubin

Petersburg Nuclear Physics Institute, Gatchina (St. Petersburg), Russia

L. Chtchipounov, V. Golovtcov, Y. Ivanov, V. Kim ${ }^{40}$, E. Kuznetsova ${ }^{41}$, P. Levchenko, V. Murzin, V. Oreshkin, I. Smirnov, D. Sosnov, V. Sulimov, L. Uvarov, A. Vorobyev

Institute for Nuclear Research, Moscow, Russia

Yu. Andreev, A. Dermenev, S. Gninenko, N. Golubev, A. Karneyeu, M. Kirsanov, N. Krasnikov, A. Pashenkov, D. Tlisov, A. Toropin

Institute for Theoretical and Experimental Physics named by A.I. Alikhanov of NRC 'Kurchatov Institute', Moscow, Russia

V. Epshteyn, V. Gavrilov, N. Lychkovskaya, A. Nikitenko ${ }^{42}$, V. Popov, I. Pozdnyakov, G. Safronov, A. Spiridonov, A. Stepennov, M. Toms, E. Vlasov, A. Zhokin

Moscow Institute of Physics and Technology, Moscow, Russia

T. Aushev

National Research Nuclear University 'Moscow Engineering Physics Institute' (MEPhI), Moscow, Russia

M. Chadeeva ${ }^{43}$, P. Parygin, D. Philippov, E. Popova, V. Rusinov

P.N. Lebedev Physical Institute, Moscow, Russia

V. Andreev, M. Azarkin, I. Dremin, M. Kirakosyan, A. Terkulov 
Skobeltsyn Institute of Nuclear Physics, Lomonosov Moscow State University, Moscow, Russia

A. Belyaev, E. Boos, V. Bunichev, M. Dubinin ${ }^{44}$, L. Dudko, A. Gribushin, V. Klyukhin, O. Kodolova, I. Lokhtin, S. Obraztsov, M. Perfilov, V. Savrin, A. Snigirev

Novosibirsk State University (NSU), Novosibirsk, Russia

A. Barnyakov ${ }^{45}$, V. Blinov ${ }^{45}$, T. Dimova ${ }^{45}$, L. Kardapoltsev ${ }^{45}$, Y. Skovpen ${ }^{45}$

Institute for High Energy Physics of National Research Centre 'Kurchatov Institute', Protvino, Russia

I. Azhgirey, I. Bayshev, S. Bitioukov, V. Kachanov, D. Konstantinov, P. Mandrik,

V. Petrov, R. Ryutin, S. Slabospitskii, A. Sobol, S. Troshin, N. Tyurin, A. Uzunian,

A. Volkov

National Research Tomsk Polytechnic University, Tomsk, Russia

A. Babaev, A. Iuzhakov, V. Okhotnikov

Tomsk State University, Tomsk, Russia

V. Borchsh, V. Ivanchenko, E. Tcherniaev

University of Belgrade: Faculty of Physics and VINCA Institute of Nuclear Sciences

P. Adzic ${ }^{46}$, P. Cirkovic, M. Dordevic, P. Milenovic, J. Milosevic, M. Stojanovic

Centro de Investigaciones Energéticas Medioambientales y Tecnológicas (CIEMAT), Madrid, Spain

M. Aguilar-Benitez, J. Alcaraz Maestre, A. Álvarez Fernández, I. Bachiller, M. Barrio Luna, CristinaF. Bedoya, J.A. Brochero Cifuentes, C.A. Carrillo Montoya, M. Cepeda, M. Cerrada, N. Colino, B. De La Cruz, A. Delgado Peris, J.P. Fernández Ramos, J. Flix, M.C. Fouz, O. Gonzalez Lopez, S. Goy Lopez, J.M. Hernandez, M.I. Josa, D. Moran, Á. Navarro Tobar, A. Pérez-Calero Yzquierdo, J. Puerta Pelayo, I. Redondo, L. Romero, S. Sánchez Navas, M.S. Soares, A. Triossi, C. Willmott

Universidad Autónoma de Madrid, Madrid, Spain

C. Albajar, J.F. de Trocóniz, R. Reyes-Almanza

Universidad de Oviedo, Instituto Universitario de Ciencias y Tecnologías Espaciales de Asturias (ICTEA), Oviedo, Spain

B. Alvarez Gonzalez, J. Cuevas, C. Erice, J. Fernandez Menendez, S. Folgueras, I. Gonzalez Caballero, J.R. González Fernández, E. Palencia Cortezon, V. Rodríguez Bouza, S. Sanchez Cruz

Instituto de Física de Cantabria (IFCA), CSIC-Universidad de Cantabria, Santander, Spain

I.J. Cabrillo, A. Calderon, B. Chazin Quero, J. Duarte Campderros, M. Fernandez, P.J. Fernández Manteca, A. García Alonso, G. Gomez, C. Martinez Rivero, P. Martinez Ruiz del Arbol, F. Matorras, J. Piedra Gomez, C. Prieels, T. Rodrigo, A. Ruiz-Jimeno, L. Russo ${ }^{47}$, L. Scodellaro, I. Vila, J.M. Vizan Garcia 


\section{University of Colombo, Colombo, Sri Lanka}

K. Malagalage

\section{University of Ruhuna, Department of Physics, Matara, Sri Lanka}

W.G.D. Dharmaratna, N. Wickramage

\section{CERN, European Organization for Nuclear Research, Geneva, Switzerland}

D. Abbaneo, B. Akgun, E. Auffray, G. Auzinger, J. Baechler, P. Baillon, A.H. Ball, D. Barney, J. Bendavid, M. Bianco, A. Bocci, P. Bortignon, E. Bossini, C. Botta, E. Brondolin, T. Camporesi, A. Caratelli, G. Cerminara, E. Chapon, G. Cucciati, D. d'Enterria, A. Dabrowski, N. Daci, V. Daponte, A. David, O. Davignon, A. De Roeck, M. Deile, M. Dobson, M. Dünser, N. Dupont, A. Elliott-Peisert, N. Emriskova, F. Fallavollita ${ }^{48}$, D. Fasanella, S. Fiorendi, G. Franzoni, J. Fulcher, W. Funk, S. Giani, D. Gigi, K. Gill, F. Glege, L. Gouskos, M. Gruchala, M. Guilbaud, D. Gulhan, J. Hegeman, C. Heidegger, Y. Iiyama, V. Innocente, T. James, P. Janot, O. Karacheban ${ }^{20}$, J. Kaspar, J. Kieseler, M. Krammer ${ }^{1}$, N. Kratochwil, C. Lange, P. Lecoq, C. Lourenço, L. Malgeri, M. Mannelli, A. Massironi, F. Meijers, S. Mersi, E. Meschi, F. Moortgat, M. Mulders, J. Ngadiuba, J. Niedziela, S. Nourbakhsh, S. Orfanelli, L. Orsini, F. Pantaleo ${ }^{17}$, L. Pape, E. Perez, M. Peruzzi, A. Petrilli, G. Petrucciani, A. Pfeiffer, M. Pierini, F.M. Pitters, D. Rabady, A. Racz, M. Rieger, M. Rovere, H. Sakulin, J. Salfeld-Nebgen, C. Schäfer, C. Schwick, M. Selvaggi, A. Sharma, P. Silva, W. Snoeys, P. Sphicas ${ }^{49}$, J. Steggemann, S. Summers, V.R. Tavolaro, D. Treille, A. Tsirou, G.P. Van Onsem, A. Vartak, M. Verzetti, W.D. Zeuner

\section{Paul Scherrer Institut, Villigen, Switzerland}

L. Caminada ${ }^{50}$, K. Deiters, W. Erdmann, R. Horisberger, Q. Ingram, H.C. Kaestli, D. Kotlinski, U. Langenegger, T. Rohe, S.A. Wiederkehr

ETH Zurich - Institute for Particle Physics and Astrophysics (IPA), Zurich, Switzerland

M. Backhaus, P. Berger, N. Chernyavskaya, G. Dissertori, M. Dittmar, M. Donegà, C. Dorfer, T.A. Gómez Espinosa, C. Grab, D. Hits, W. Lustermann, R.A. Manzoni, M.T. Meinhard, F. Micheli, P. Musella, F. Nessi-Tedaldi, F. Pauss, G. Perrin, L. Perrozzi, S. Pigazzini, M.G. Ratti, M. Reichmann, C. Reissel, T. Reitenspiess, B. Ristic, D. Ruini, D.A. Sanz Becerra, M. Schönenberger, L. Shchutska, M.L. Vesterbacka Olsson, R. Wallny, D.H. Zhu

\section{Universität Zürich, Zurich, Switzerland}

T.K. Aarrestad, C. Amsler ${ }^{51}$, D. Brzhechko, M.F. Canelli, A. De Cosa, R. Del Burgo, B. Kilminster, S. Leontsinis, V.M. Mikuni, I. Neutelings, G. Rauco, P. Robmann, K. Schweiger, C. Seitz, Y. Takahashi, S. Wertz, A. Zucchetta

\section{National Central University, Chung-Li, Taiwan}

T.H. Doan, C.M. Kuo, W. Lin, A. Roy, S.S. Yu

\section{National Taiwan University (NTU), Taipei, Taiwan}

P. Chang, Y. Chao, K.F. Chen, P.H. Chen, W.-S. Hou, Y.y. Li, R.-S. Lu, E. Paganis, A. Psallidas, A. Steen 
Chulalongkorn University, Faculty of Science, Department of Physics, Bangkok, Thailand

B. Asavapibhop, C. Asawatangtrakuldee, N. Srimanobhas, N. Suwonjandee

Çukurova University, Physics Department, Science and Art Faculty, Adana, Turkey

A. Bat, F. Boran, A. Celik ${ }^{52}$, S. Damarseckin ${ }^{53}$, Z.S. Demiroglu, F. Dolek, C. Dozen ${ }^{54}$, I. Dumanoglu, G. Gokbulut, EmineGurpinar Guler ${ }^{55}$, Y. Guler, I. Hos ${ }^{56}$, C. Isik, E.E. Kangal ${ }^{57}$, O. Kara, A. Kayis Topaksu, U. Kiminsu, G. Onengut, K. Ozdemir ${ }^{58}$, S. Ozturk ${ }^{59}$, A.E. Simsek, U.G. Tok, S. Turkcapar, I.S. Zorbakir, C. Zorbilmez

Middle East Technical University, Physics Department, Ankara, Turkey B. Isildak ${ }^{60}$, G. Karapinar ${ }^{61}$, M. Yalvac

Bogazici University, Istanbul, Turkey

I.O. Atakisi, E. Gülmez, M. Kaya ${ }^{62}$, O. Kaya ${ }^{63}$, Ö. Özçelik, S. Tekten, E.A. Yetkin ${ }^{64}$

Istanbul Technical University, Istanbul, Turkey

A. Cakir, K. Cankocak, Y. Komurcu, S. Sen ${ }^{65}$

Istanbul University, Istanbul, Turkey

S. $\operatorname{Cerci}^{66}$, B. Kaynak, S. Ozkorucuklu, D. Sunar Cerci ${ }^{66}$

Institute for Scintillation Materials of National Academy of Science of Ukraine, Kharkov, Ukraine

B. Grynyov

National Scientific Center, Kharkov Institute of Physics and Technology, Kharkov, Ukraine

L. Levchuk

University of Bristol, Bristol, United Kingdom

E. Bhal, S. Bologna, J.J. Brooke, D. Burns ${ }^{67}$, E. Clement, D. Cussans, H. Flacher, J. Goldstein, G.P. Heath, H.F. Heath, L. Kreczko, B. Krikler, S. Paramesvaran, B. Penning, T. Sakuma, S. Seif El Nasr-Storey, V.J. Smith, J. Taylor, A. Titterton

Rutherford Appleton Laboratory, Didcot, United Kingdom

K.W. Bell, A. Belyaev ${ }^{68}$, C. Brew, R.M. Brown, D.J.A. Cockerill, J.A. Coughlan, K. Harder, S. Harper, J. Linacre, K. Manolopoulos, D.M. Newbold, E. Olaiya, D. Petyt, T. Reis, T. Schuh, C.H. Shepherd-Themistocleous, A. Thea, I.R. Tomalin, T. Williams

Imperial College, London, United Kingdom

R. Bainbridge, P. Bloch, J. Borg, S. Breeze, O. Buchmuller, A. Bundock, GurpreetSingh CHAHAL ${ }^{69}$, D. Colling, P. Dauncey, G. Davies, M. Della Negra, R. Di Maria, P. Everaerts, G. Hall, G. Iles, M. Komm, L. Lyons, A.-M. Magnan, S. Malik, A. Martelli, V. Milosevic, A. Morton, J. Nash ${ }^{70}$, V. Palladino, M. Pesaresi, D.M. Raymond, A. Richards, A. Rose, E. Scott, C. Seez, A. Shtipliyski, M. Stoye, T. Strebler, A. Tapper, K. Uchida, T. Virdee ${ }^{17}$, N. Wardle, D. Winterbottom, A.G. Zecchinelli, S.C. Zenz 
Brunel University, Uxbridge, United Kingdom

J.E. Cole, P.R. Hobson, A. Khan, P. Kyberd, C.K. Mackay, I.D. Reid, L. Teodorescu, S. Zahid

Baylor University, Waco, U.S.A.

K. Call, B. Caraway, J. Dittmann, K. Hatakeyama, C. Madrid, B. McMaster, N. Pastika, C. Smith

Catholic University of America, Washington, DC, U.S.A.

R. Bartek, A. Dominguez, R. Uniyal, A.M. Vargas Hernandez

The University of Alabama, Tuscaloosa, U.S.A.

A. Buccilli, S.I. Cooper, C. Henderson, P. Rumerio, C. West

Boston University, Boston, U.S.A.

A. Albert, D. Arcaro, Z. Demiragli, D. Gastler, C. Richardson, J. Rohlf, D. Sperka, I. Suarez, L. Sulak, D. Zou

Brown University, Providence, U.S.A.

G. Benelli, B. Burkle, X. Coubez ${ }^{18}$, D. Cutts, Y.t. Duh, M. Hadley, U. Heintz, J.M. $\operatorname{Hogan}^{71}$, K.H.M. Kwok, E. Laird, G. Landsberg, K.T. Lau, J. Lee, M. Narain, S. Sagir ${ }^{72}$, R. Syarif, E. Usai, W.Y. Wong, D. Yu, W. Zhang

University of California, Davis, Davis, U.S.A.

R. Band, C. Brainerd, R. Breedon, M. Calderon De La Barca Sanchez, M. Chertok, J. Conway, R. Conway, P.T. Cox, R. Erbacher, C. Flores, G. Funk, F. Jensen, W. Ko ${ }^{\dagger}$, O. Kukral, R. Lander, M. Mulhearn, D. Pellett, J. Pilot, M. Shi, D. Taylor, K. Tos, M. Tripathi, Z. Wang, F. Zhang

University of California, Los Angeles, U.S.A.

M. Bachtis, C. Bravo, R. Cousins, A. Dasgupta, A. Florent, J. Hauser, M. Ignatenko, N. Mccoll, W.A. Nash, S. Regnard, D. Saltzberg, C. Schnaible, B. Stone, V. Valuev

University of California, Riverside, Riverside, U.S.A.

K. Burt, Y. Chen, R. Clare, J.W. Gary, S.M.A. Ghiasi Shirazi, G. Hanson, G. Karapostoli, O.R. Long, M. Olmedo Negrete, M.I. Paneva, W. Si, L. Wang, S. Wimpenny, B.R. Yates, Y. Zhang

University of California, San Diego, La Jolla, U.S.A.

J.G. Branson, P. Chang, S. Cittolin, S. Cooperstein, N. Deelen, M. Derdzinski, R. Gerosa, D. Gilbert, B. Hashemi, D. Klein, V. Krutelyov, J. Letts, M. Masciovecchio, S. May, S. Padhi, M. Pieri, V. Sharma, M. Tadel, F. Würthwein, A. Yagil, G. Zevi Della Porta

University of California, Santa Barbara - Department of Physics, Santa Barbara, U.S.A.

N. Amin, R. Bhandari, C. Campagnari, M. Citron, V. Dutta, M. Franco Sevilla, J. Incandela, B. Marsh, H. Mei, A. Ovcharova, H. Qu, J. Richman, U. Sarica, D. Stuart, S. Wang 
California Institute of Technology, Pasadena, U.S.A.

D. Anderson, A. Bornheim, O. Cerri, I. Dutta, J.M. Lawhorn, N. Lu, J. Mao, H.B. Newman, T.Q. Nguyen, J. Pata, M. Spiropulu, J.R. Vlimant, S. Xie, Z. Zhang, R.Y. Zhu

Carnegie Mellon University, Pittsburgh, U.S.A.

M.B. Andrews, T. Ferguson, T. Mudholkar, M. Paulini, M. Sun, I. Vorobiev, M. Weinberg

University of Colorado Boulder, Boulder, U.S.A.

J.P. Cumalat, W.T. Ford, E. MacDonald, T. Mulholland, R. Patel, A. Perloff, K. Stenson, K.A. Ulmer, S.R. Wagner

Cornell University, Ithaca, U.S.A.

J. Alexander, Y. Cheng, J. Chu, A. Datta, A. Frankenthal, K. Mcdermott, J.R. Patterson,

D. Quach, A. Ryd, S.M. Tan, Z. Tao, J. Thom, P. Wittich, M. Zientek

Fermi National Accelerator Laboratory, Batavia, U.S.A.

S. Abdullin, M. Albrow, M. Alyari, G. Apollinari, A. Apresyan, A. Apyan, S. Banerjee, L.A.T. Bauerdick, A. Beretvas, D. Berry, J. Berryhill, P.C. Bhat, K. Burkett, J.N. Butler, A. Canepa, G.B. Cerati, H.W.K. Cheung, F. Chlebana, M. Cremonesi, J. Duarte, V.D. Elvira, J. Freeman, Z. Gecse, E. Gottschalk, L. Gray, D. Green, S. Grünendahl, O. Gutsche, AllisonReinsvold Hall, J. Hanlon, R.M. Harris, S. Hasegawa, R. Heller, J. Hirschauer, B. Jayatilaka, S. Jindariani, M. Johnson, U. Joshi, T. Klijnsma, B. Klima, M.J. Kortelainen, B. Kreis, S. Lammel, J. Lewis, D. Lincoln, R. Lipton, M. Liu, T. Liu, J. Lykken, K. Maeshima, J.M. Marraffino, D. Mason, P. McBride, P. Merkel, S. Mrenna, S. Nahn, V. O'Dell, V. Papadimitriou, K. Pedro, C. Pena, G. Rakness, F. Ravera, L. Ristori, B. Schneider, E. Sexton-Kennedy, N. Smith, A. Soha, W.J. Spalding, L. Spiegel, S. Stoynev, J. Strait, N. Strobbe, L. Taylor, S. Tkaczyk, N.V. Tran, L. Uplegger, E.W. Vaandering, C. Vernieri, R. Vidal, M. Wang, H.A. Weber

University of Florida, Gainesville, U.S.A.

D. Acosta, P. Avery, D. Bourilkov, A. Brinkerhoff, L. Cadamuro, V. Cherepanov, F. Errico, R.D. Field, S.V. Gleyzer, D. Guerrero, B.M. Joshi, M. Kim, J. Konigsberg, A. Korytov, K.H. Lo, K. Matchev, N. Menendez, G. Mitselmakher, D. Rosenzweig, K. Shi, J. Wang, S. Wang, X. Zuo

\section{Florida International University, Miami, U.S.A.}

Y.R. Joshi

Florida State University, Tallahassee, U.S.A.

T. Adams, A. Askew, S. Hagopian, V. Hagopian, K.F. Johnson, R. Khurana, T. Kolberg,

G. Martinez, T. Perry, H. Prosper, C. Schiber, R. Yohay, J. Zhang

Florida Institute of Technology, Melbourne, U.S.A.

M.M. Baarmand, M. Hohlmann, D. Noonan, M. Rahmani, M. Saunders, F. Yumiceva

University of Illinois at Chicago (UIC), Chicago, U.S.A.

M.R. Adams, L. Apanasevich, R.R. Betts, R. Cavanaugh, X. Chen, S. Dittmer, O. Evdokimov, C.E. Gerber, D.A. Hangal, D.J. Hofman, C. Mills, T. Roy, M.B. Tonjes, N. Varelas, J. Viinikainen, H. Wang, X. Wang, Z. Wu 
The University of Iowa, Iowa City, U.S.A.

M. Alhusseini, B. Bilki ${ }^{55}$, K. Dilsiz ${ }^{73}$, S. Durgut, R.P. Gandrajula, M. Haytmyradov, V. Khristenko, O.K. Köseyan, J.-P. Merlo, A. Mestvirishvilii ${ }^{74}$, A. Moeller, J. Nachtman, H. Ogul ${ }^{75}$, Y. Onel, F. Ozok ${ }^{76}$, A. Penzo, C. Snyder, E. Tiras, J. Wetzel

Johns Hopkins University, Baltimore, U.S.A.

B. Blumenfeld, A. Cocoros, N. Eminizer, A.V. Gritsan, W.T. Hung, S. Kyriacou, P. Maksimovic, J. Roskes, M. Swartz

The University of Kansas, Lawrence, U.S.A.

C. Baldenegro Barrera, P. Baringer, A. Bean, S. Boren, J. Bowen, A. Bylinkin, T. Isidori, S. Khalil, J. King, G. Krintiras, A. Kropivnitskaya, C. Lindsey, D. Majumder, W. Mcbrayer, N. Minafra, M. Murray, C. Rogan, C. Royon, S. Sanders, E. Schmitz, J.D. Tapia Takaki, Q. Wang, J. Williams, G. Wilson

Kansas State University, Manhattan, U.S.A.

S. Duric, A. Ivanov, K. Kaadze, D. Kim, Y. Maravin, D.R. Mendis, T. Mitchell, A. Modak, A. Mohammadi

Lawrence Livermore National Laboratory, Livermore, U.S.A.

F. Rebassoo, D. Wright

University of Maryland, College Park, U.S.A.

A. Baden, O. Baron, A. Belloni, S.C. Eno, Y. Feng, N.J. Hadley, S. Jabeen, G.Y. Jeng, R.G. Kellogg, A.C. Mignerey, S. Nabili, F. Ricci-Tam, M. Seidel, Y.H. Shin, A. Skuja, S.C. Tonwar, K. Wong

Massachusetts Institute of Technology, Cambridge, U.S.A.

D. Abercrombie, B. Allen, A. Baty, R. Bi, S. Brandt, W. Busza, I.A. Cali, M. D'Alfonso, G. Gomez Ceballos, M. Goncharov, P. Harris, D. Hsu, M. Hu, M. Klute, D. Kovalskyi, Y.-J. Lee, P.D. Luckey, B. Maier, A.C. Marini, C. Mcginn, C. Mironov, S. Narayanan, X. Niu, C. Paus, D. Rankin, C. Roland, G. Roland, Z. Shi, G.S.F. Stephans, K. Sumorok, K. Tatar, D. Velicanu, J. Wang, T.W. Wang, B. Wyslouch

University of Minnesota, Minneapolis, U.S.A.

R.M. Chatterjee, A. Evans, S. Guts ${ }^{\dagger}$, P. Hansen, J. Hiltbrand, Sh. Jain, Y. Kubota, Z. Lesko, J. Mans, M. Revering, R. Rusack, R. Saradhy, N. Schroeder, M.A. Wadud

University of Mississippi, Oxford, U.S.A.

J.G. Acosta, S. Oliveros

University of Nebraska-Lincoln, Lincoln, U.S.A.

K. Bloom, S. Chauhan, D.R. Claes, C. Fangmeier, L. Finco, F. Golf, R. Kamalieddin, I. Kravchenko, J.E. Siado, G.R. Snow ${ }^{\dagger}$, B. Stieger, W. Tabb

State University of New York at Buffalo, Buffalo, U.S.A.

G. Agarwal, C. Harrington, I. Iashvili, A. Kharchilava, C. McLean, D. Nguyen, A. Parker, J. Pekkanen, S. Rappoccio, B. Roozbahani 
Northeastern University, Boston, U.S.A.

G. Alverson, E. Barberis, C. Freer, Y. Haddad, A. Hortiangtham, G. Madigan, B. Marzocchi, D.M. Morse, T. Orimoto, L. Skinnari, A. Tishelman-Charny, T. Wamorkar, B. Wang, A. Wisecarver, D. Wood

Northwestern University, Evanston, U.S.A.

S. Bhattacharya, J. Bueghly, A. Gilbert, T. Gunter, K.A. Hahn, N. Odell, M.H. Schmitt, K. Sung, M. Trovato, M. Velasco

University of Notre Dame, Notre Dame, U.S.A.

R. Bucci, N. Dev, R. Goldouzian, M. Hildreth, K. Hurtado Anampa, C. Jessop, D.J. Karmgard, K. Lannon, W. Li, N. Loukas, N. Marinelli, I. Mcalister, F. Meng, Y. Musienko ${ }^{38}$, R. Ruchti, P. Siddireddy, G. Smith, S. Taroni, M. Wayne, A. Wightman, M. Wolf, A. Woodard

The Ohio State University, Columbus, U.S.A.

J. Alimena, B. Bylsma, L.S. Durkin, B. Francis, C. Hill, W. Ji, A. Lefeld, T.Y. Ling, B.L. Winer

Princeton University, Princeton, U.S.A.

G. Dezoort, P. Elmer, J. Hardenbrook, N. Haubrich, S. Higginbotham, A. Kalogeropoulos, S. Kwan, D. Lange, M.T. Lucchini, J. Luo, D. Marlow, K. Mei, I. Ojalvo, J. Olsen, C. Palmer, P. Piroué, D. Stickland, C. Tully

University of Puerto Rico, Mayaguez, U.S.A.

S. Malik, S. Norberg

Purdue University, West Lafayette, U.S.A.

A. Barker, V.E. Barnes, S. Das, L. Gutay, M. Jones, A.W. Jung, A. Khatiwada, B. Mahakud, D.H. Miller, G. Negro, N. Neumeister, C.C. Peng, S. Piperov, H. Qiu, J.F. Schulte, N. Trevisani, F. Wang, R. Xiao, W. Xie

Purdue University Northwest, Hammond, U.S.A.

T. Cheng, J. Dolen, N. Parashar

Rice University, Houston, U.S.A.

U. Behrens, K.M. Ecklund, S. Freed, F.J.M. Geurts, M. Kilpatrick, Arun Kumar, W. Li, B.P. Padley, R. Redjimi, J. Roberts, J. Rorie, W. Shi, A.G. Stahl Leiton, Z. Tu, A. Zhang

University of Rochester, Rochester, U.S.A.

A. Bodek, P. de Barbaro, R. Demina, J.L. Dulemba, C. Fallon, T. Ferbel, M. Galanti, A. Garcia-Bellido, O. Hindrichs, A. Khukhunaishvili, E. Ranken, R. Taus

Rutgers, The State University of New Jersey, Piscataway, U.S.A.

B. Chiarito, J.P. Chou, A. Gandrakota, Y. Gershtein, E. Halkiadakis, A. Hart, M. Heindl,

E. Hughes, S. Kaplan, I. Laflotte, A. Lath, R. Montalvo, K. Nash, M. Osherson, H. Saka,

S. Salur, S. Schnetzer, S. Somalwar, R. Stone, S. Thomas 
University of Tennessee, Knoxville, U.S.A.

H. Acharya, A.G. Delannoy, S. Spanier

Texas A\&M University, College Station, U.S.A.

O. Bouhali ${ }^{77}$, M. Dalchenko, M. De Mattia, A. Delgado, S. Dildick, R. Eusebi, J. Gilmore,

T. Huang, T. Kamon ${ }^{78}$, H. Kim, S. Luo, S. Malhotra, D. Marley, R. Mueller, D. Overton,

L. Perniè, D. Rathjens, A. Safonov

Texas Tech University, Lubbock, U.S.A.

N. Akchurin, J. Damgov, F. De Guio, V. Hegde, S. Kunori, K. Lamichhane, S.W. Lee, T. Mengke, S. Muthumuni, T. Peltola, S. Undleeb, I. Volobouev, Z. Wang, A. Whitbeck

Vanderbilt University, Nashville, U.S.A.

S. Greene, A. Gurrola, R. Janjam, W. Johns, C. Maguire, A. Melo, H. Ni, K. Padeken, F. Romeo, P. Sheldon, S. Tuo, J. Velkovska, M. Verweij

University of Virginia, Charlottesville, U.S.A.

M.W. Arenton, P. Barria, B. Cox, G. Cummings, J. Hakala, R. Hirosky, M. Joyce, A. Ledovskoy, C. Neu, B. Tannenwald, Y. Wang, E. Wolfe, F. Xia

Wayne State University, Detroit, U.S.A.

R. Harr, P.E. Karchin, N. Poudyal, J. Sturdy, P. Thapa

University of Wisconsin - Madison, Madison, WI, U.S.A.

T. Bose, J. Buchanan, C. Caillol, D. Carlsmith, S. Dasu, I. De Bruyn, L. Dodd, C. Galloni, H. He, M. Herndon, A. Hervé, U. Hussain, A. Lanaro, A. Loeliger, K. Long, R. Loveless, J. Madhusudanan Sreekala, D. Pinna, T. Ruggles, A. Savin, V. Sharma, W.H. Smith, D. Teague, S. Trembath-reichert

$\dagger$ : Deceased

1: Also at Vienna University of Technology, Vienna, Austria

2: Also at IRFU, CEA, Université Paris-Saclay, Gif-sur-Yvette, France

3: Also at Universidade Estadual de Campinas, Campinas, Brazil

4: Also at Federal University of Rio Grande do Sul, Porto Alegre, Brazil

5: Also at UFMS, Nova Andradina, Brazil

6: Also at Universidade Federal de Pelotas, Pelotas, Brazil

7: Also at Université Libre de Bruxelles, Bruxelles, Belgium

8: Also at University of Chinese Academy of Sciences, Beijing, China

9: Also at Institute for Theoretical and Experimental Physics named by A.I. Alikhanov of NRC 'Kurchatov Institute', Moscow, Russia

10: Also at Joint Institute for Nuclear Research, Dubna, Russia

11: Also at Suez University, Suez, Egypt

12: Now at British University in Egypt, Cairo, Egypt

13: Also at Purdue University, West Lafayette, U.S.A.

14: Also at Université de Haute Alsace, Mulhouse, France

15: Also at Tbilisi State University, Tbilisi, Georgia

16: Also at Erzincan Binali Yildirim University, Erzincan, Turkey

17: Also at CERN, European Organization for Nuclear Research, Geneva, Switzerland 
18: Also at RWTH Aachen University, III. Physikalisches Institut A, Aachen, Germany

19: Also at University of Hamburg, Hamburg, Germany

20: Also at Brandenburg University of Technology, Cottbus, Germany

21: Also at Institute of Physics, University of Debrecen, Debrecen, Hungary, Debrecen, Hungary

22: Also at Institute of Nuclear Research ATOMKI, Debrecen, Hungary

23: Also at MTA-ELTE Lendület CMS Particle and Nuclear Physics Group, Eötvös Loránd University, Budapest, Hungary, Budapest, Hungary

24: Also at IIT Bhubaneswar, Bhubaneswar, India, Bhubaneswar, India

25: Also at Institute of Physics, Bhubaneswar, India

26: Also at Shoolini University, Solan, India

27: Also at University of Hyderabad, Hyderabad, India

28: Also at University of Visva-Bharati, Santiniketan, India

29: Also at Isfahan University of Technology, Isfahan, Iran

30: Now at INFN Sezione di Bari ${ }^{a}$, Università di Bari ${ }^{b}$, Politecnico di Bari ${ }^{c}$, Bari, Italy

31: Also at Italian National Agency for New Technologies, Energy and Sustainable Economic Development, Bologna, Italy

32: Also at Centro Siciliano di Fisica Nucleare e di Struttura Della Materia, Catania, Italy

33: Also at Scuola Normale e Sezione dell'INFN, Pisa, Italy

34: Also at Riga Technical University, Riga, Latvia, Riga, Latvia

35: Also at Malaysian Nuclear Agency, MOSTI, Kajang, Malaysia

36: Also at Consejo Nacional de Ciencia y Tecnología, Mexico City, Mexico

37: Also at Warsaw University of Technology, Institute of Electronic Systems, Warsaw, Poland

38: Also at Institute for Nuclear Research, Moscow, Russia

39: Now at National Research Nuclear University 'Moscow Engineering Physics Institute' (MEPhI), Moscow, Russia

40: Also at St. Petersburg State Polytechnical University, St. Petersburg, Russia

41: Also at University of Florida, Gainesville, U.S.A.

42: Also at Imperial College, London, United Kingdom

43: Also at P.N. Lebedev Physical Institute, Moscow, Russia

44: Also at California Institute of Technology, Pasadena, U.S.A.

45: Also at Budker Institute of Nuclear Physics, Novosibirsk, Russia

46: Also at Faculty of Physics, University of Belgrade, Belgrade, Serbia

47: Also at Università degli Studi di Siena, Siena, Italy

48: Also at INFN Sezione di Pavia ${ }^{a}$, Università di Pavia ${ }^{b}$, Pavia, Italy, Pavia, Italy

49: Also at National and Kapodistrian University of Athens, Athens, Greece

50: Also at Universität Zürich, Zurich, Switzerland

51: Also at Stefan Meyer Institute for Subatomic Physics, Vienna, Austria, Vienna, Austria

52: Also at Burdur Mehmet Akif Ersoy University, BURDUR, Turkey

53: Also at Şırnak University, Sirnak, Turkey

54: Also at Department of Physics, Tsinghua University, Beijing, China, Beijing, China

55: Also at Beykent University, Istanbul, Turkey, Istanbul, Turkey

56: Also at Istanbul Aydin University, Application and Research Center for Advanced Studies (App. \& Res. Cent. for Advanced Studies), Istanbul, Turkey

57: Also at Mersin University, Mersin, Turkey

58: Also at Piri Reis University, Istanbul, Turkey

59: Also at Gaziosmanpasa University, Tokat, Turkey

60: Also at Ozyegin University, Istanbul, Turkey

61: Also at Izmir Institute of Technology, Izmir, Turkey 
62: Also at Marmara University, Istanbul, Turkey

63: Also at Kafkas University, Kars, Turkey

64: Also at Istanbul Bilgi University, Istanbul, Turkey

65: Also at Hacettepe University, Ankara, Turkey

66: Also at Adiyaman University, Adiyaman, Turkey

67: Also at Vrije Universiteit Brussel, Brussel, Belgium

68: Also at School of Physics and Astronomy, University of Southampton, Southampton, United Kingdom

69: Also at IPPP Durham University, Durham, United Kingdom

70: Also at Monash University, Faculty of Science, Clayton, Australia

71: Also at Bethel University, St. Paul, Minneapolis, U.S.A., St. Paul, U.S.A.

72: Also at Karamanoğlu Mehmetbey University, Karaman, Turkey

73: Also at Bingol University, Bingol, Turkey

74: Also at Georgian Technical University, Tbilisi, Georgia

75: Also at Sinop University, Sinop, Turkey

76: Also at Mimar Sinan University, Istanbul, Istanbul, Turkey

77: Also at Texas A\&M University at Qatar, Doha, Qatar

78: Also at Kyungpook National University, Daegu, Korea, Daegu, Korea 